\title{
The deep Chandra survey in the SDSS J1030+0524 field ${ }^{\star}$
}

\author{
R. Nanni ${ }^{1,2,3}$, R. Gilli ${ }^{1}$, C. Vignali ${ }^{2,1}$, M. Mignoli ${ }^{1}$, A. Peca ${ }^{1,4}$, S. Marchesi ${ }^{1,5}$, M. Annunziatella ${ }^{6}$, M. Brusa ${ }^{2}$, \\ F. Calura ${ }^{1}$, N. Cappelluti ${ }^{4,7,8}$, M. Chiaberge ${ }^{9,10}$, A. Comastri ${ }^{1}$, K. Iwasawa ${ }^{11,12}$, G. Lanzuisi ${ }^{1}$, E. Liuzzo ${ }^{13}$, \\ D. Marchesini ${ }^{6}$, I. Prandoni ${ }^{13}$, P. Tozzi ${ }^{14}$, F. Vito ${ }^{15,16}$, G. Zamorani ${ }^{1}$, and C. Norman ${ }^{9,10}$ \\ 1 INAF - Osservatorio di Astrofisica e Scienza dello Spazio di Bologna, Via Gobetti 93/3, 40129 Bologna, Italy \\ e-mail: riccardo.nanni7@unibo.it \\ 2 Dipartimento di Fisica e Astronomia, Università degli Studi di Bologna, Via Gobetti 93/2, 40129 Bologna, Italy \\ 3 Department of Physics, University of California, Santa Barbara, CA 93106-9530, USA \\ ${ }^{4}$ Physics Department, University of Miami, Coral Gables, FL 33124, USA \\ 5 Department of Physics and Astronomy, Clemson University, Kinard Lab of Physics, Clemson, SC 29634, USA \\ ${ }^{6}$ Physics Department, Tufts University, 574 Boston Avenue, Medford, MA 02155, USA \\ 7 Yale Center for Astronomy and Astrophysics, PO Box 208121, New Haven, CT 06520, USA \\ 8 Department of Physics, Yale University, PO Box 208121, New Haven, CT 06520, USA \\ 9 Space Telescope Science Institute, 3700 San Martin Dr., Baltimore, MD 21210, USA \\ 10 Johns Hopkins University, 3400 N. Charles Street, Baltimore, MD 21218, USA \\ 11 Institut de Ciències del Cosmos (ICCUB), Universitat de Barcelona (IEEC-UB), Martí i Franquès, 1, 08028 Barcelona, Spain \\ 125 ICREA, Pg. Lluís Companys 23, 08010 Barcelona, Spain \\ 13 INAF - Istituto di Radioastronomia, Via Gobetti 101, 40129 Bologna, Italy \\ 14 INAF, Osservatorio Astrofisico di Arcetri, Largo E. Fermi 5, 50125 Firenze, Italy \\ 15 Instituto de Astrofisica and Centro de Astroingenieria, Facultad de Fisica, Pontificia Universidad Catolica de Chile, Casilla 306 , \\ Santiago 22, Chile \\ 16 Chinese Academy of Sciences South America Center for Astronomy, National Astronomical Observatories, CAS, \\ Beijing 100012, PR China
}

Received 9 March 2020 / Accepted 27 March 2020

\begin{abstract}
We present the X-ray source catalog for the $\sim 479 \mathrm{ks}$ Chandra exposure of the SDSS J1030+0524 field, which is centered on a region that shows the best evidence to date of an overdensity around a $z>6$ quasar, and also includes a galaxy overdensity around a Comptonthick Fanaroff-Riley type II (FRII) radio galaxy at $z=1.7$. Using wavdetect for initial source detection and ACIS Extract for source photometry and significance assessment, we create preliminary catalogs of sources that are detected in the full $(0.5-7.0 \mathrm{keV})$, soft $(0.5-2.0 \mathrm{keV})$, and hard $(2-7 \mathrm{keV})$ bands, respectively. We produce X-ray simulations that mirror our Chandra observation to filter our preliminary catalogs and achieve a completeness level of $>91 \%$ and a reliability level of $\sim 95 \%$ in each band. The catalogs in the three bands are then matched into a final main catalog of 256 unique sources. Among them, 244, 193, and 208 are detected in the full, soft, and hard bands, respectively. The Chandra observation covers a total area of $335 \mathrm{arcmin}^{2}$ and reaches flux limits over the central few square arcmins of $\sim 3 \times 10^{-16}, 6 \times 10^{-17}$, and $2 \times 10^{-16} \mathrm{erg} \mathrm{cm}^{-2} \mathrm{~s}^{-1}$ in the full, soft, and hard bands, respectively This makes J1030 field the fifth deepest extragalactic X-ray survey to date. The field is part of the Multiwavelength Survey by Yale-Chile (MUSYC), and is also covered by optical imaging data from the Large Binocular Camera (LBC) at the Large Binocular Telescope (LBT), near-infrared imaging data from the Canada France Hawaii Telescope WIRCam (CFHT/WIRCam), and Spitzer IRAC. Thanks to its dense multi-wavelength coverage, J1030 represents a legacy field for the study of large-scale structures around distant accreting supermassive black holes. Using a likelihood ratio analysis, we associate multi-band $(r, z, J$, and $4.5 \mu \mathrm{m})$ counterparts for $252(98.4 \%)$ of the 256 Chandra sources, with an estimated reliability of $95 \%$. Finally, we compute the cumulative number of sources in each X-ray band, finding that they are in general agreement with the results from the Chandra Deep Fields.
\end{abstract}

Key words. surveys - X-rays: general - catalogs - galaxies: high-redshift - quasars: supermassive black holes

\section{Introduction}

Deep X-ray surveys provide a highly efficient means to pinpoint growing black holes in active galactic nuclei (AGNs) across a wide range of redshifts, and offer insight into the demographics, physical properties, and interactions with the environment of super massive black holes (SMBHs). Furthermore, these surveys are primary tools to study the diffuse emission of clusters and

* Full Table 4 (catalog) is only available at the CDS via anonymous ftp to cdsarc.u-strasbg.fr $(130.79 .128 .5)$ or via http: //cdsarc.u-strasbg.fr/viz-bin/cat/J/A+A/637/A52 groups, as well as X-ray binaries in distant star-forming galaxies: the Chandra Deep Field-South (CDF-S; Luo et al. 2017), the Chandra Deep Field-North (CDF-N; Xue et al. 2016), the AEGIS-X survey (Nandra et al. 2015), the Chandra UKIDSS Ultra Deep Survey (X-UDS; Kocevski et al. 2018), and the COSMOS Legacy survey (Civano et al. 2016; Marchesi et al. 2016) are at present some of the main surveys used to investigate the deep X-ray Universe.

While shallow large-area surveys are essential to cover large portions of the sky whilst avoiding field-to-field variance problems and providing a global view of the most luminous X-ray 

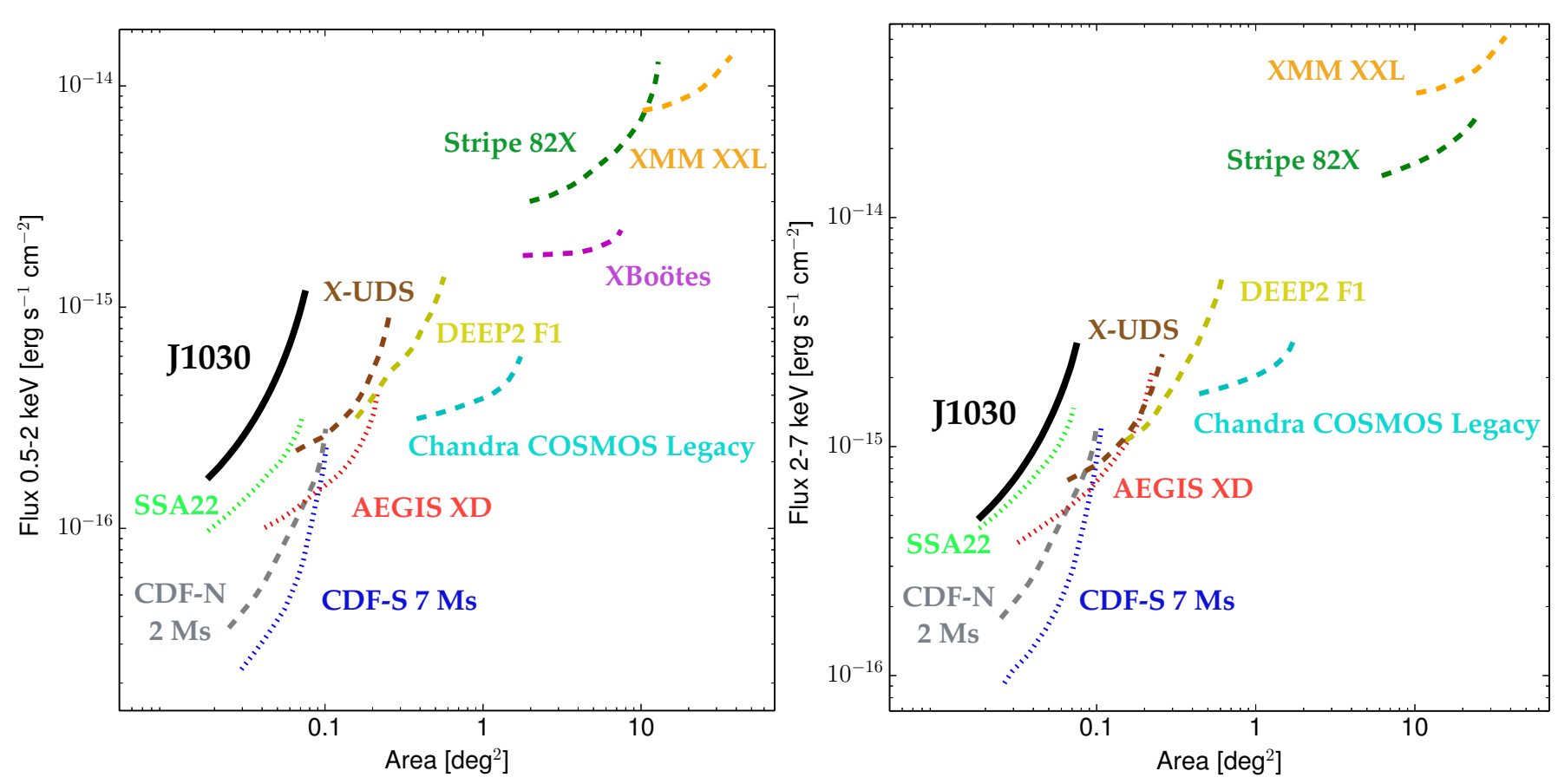

Fig. 1. Area-flux curves for different deep and moderately deep Chandra surveys in the soft (left panel) and hard (right panel) bands. Each survey has been plotted using each sensitivity curve starting from the flux corresponding to $80 \%$ of the maximum area for that survey to the flux corresponding to $20 \%$ of the total area. The reported surveys are from: this work (black line), Luo et al. (2017) (blue dotted line), Xue et al. (2016) (gray dashed line), Nandra et al. (2015) (red dotted line), Lehmer et al. (2009) (light green dotted line), Kocevski et al. (2018) (brown dashed line), Civano et al. (2016) (cyan dashed line), LaMassa et al. (2016) (dark green dotted line), Menzel et al. (2016) (orange dashed line), Goulding et al. (2012) (yellow dashed line), and Murray et al. (2005) (magenta dashed line). Despite the shorter exposure ( 400 ks), the SSA22 survey is deeper in the soft band than J1030 due to the Chandra effective area degradation (equal to $\sim 25 \%$ at $1.4 \mathrm{keV}$ ) in this band.

sources (e.g., XMM-XXL and Stripe 82X surveys; Menzel et al. 2016; LaMassa et al. 2016), deep X-ray surveys are capable of reaching extremely faint flux levels and thus earlier cosmic epochs. In addition, at a given redshift, deep surveys can probe objects with intrinsically low X-ray luminosities (which are generally more representative of the source population), including star-forming galaxies (this population is dominant at fluxes $f \leq$ $10^{-17} \mathrm{erg} \mathrm{s}^{-1} \mathrm{~cm}^{-2}$ in the $0.5-2 \mathrm{keV}$ band; Lehmer et al. 2012) and intrinsically luminous sources that are dimmed by strong nuclear obscuration (e.g., Norman et al. 2004; Comastri et al. 2011; Gilli et al. 2011).

So far, the four deepest X-ray surveys are: the CDF-S, with an exposure of $\sim 7 \mathrm{Ms}$ over an area of $484.2 \mathrm{arcmin}^{2}$ (Luo et al. 2017), the CDF-N, with an exposure of $\sim 2 \mathrm{Ms}$ over an area of $447.5 \mathrm{arcmin}^{2}$ (Xue et al. 2016), the AEGIS-X survey, with an exposure of $\sim 800 \mathrm{ks}$ over an area of $\sim 1040 \mathrm{arcmin}^{2}$ (Nandra et al. 2015), and the SSA22 survey, with an exposure of $\sim 400 \mathrm{ks}$ over an area of $\sim 330 \mathrm{arcmin}^{2}$ (Lehmer et al. 2009). These surveys achieved unprecedented X-ray sensitivity with flux limits in their inner square arcmin of $\sim 1.9,0.6,2.7 \times$ $10^{-17} \mathrm{erg} \mathrm{s}^{-1} \mathrm{~cm}^{-2}$ for CDF-S, $3.5,1.2,5.9 \times 10^{-17} \mathrm{erg} \mathrm{s}^{-1} \mathrm{~cm}^{-2}$ for CDF-N, $\sim 1.5,3.4,2.5 \times 10^{-16} \mathrm{erg} \mathrm{s}^{-1} \mathrm{~cm}^{-2}$ for AEGIS$\mathrm{X}$, and $\sim 1.7,0.6,3.0 \times 10^{-16} \mathrm{erg} \mathrm{s}^{-1} \mathrm{~cm}^{-2}$ for SSA22, in the full $(0.5-7 \mathrm{keV})$, soft $(0.5-2 \mathrm{keV})$, and hard $(2-7 \mathrm{keV})$ bands, respectively. Figure 1 shows the area-flux curves for the deepest Chandra surveys achieved so far, including the flux limits computed for J1030+0524 (hereafter J1030) in Sect. 5 .

In this paper, we present the point-source catalog derived from the $\sim 479 \mathrm{ks}$ Chandra exposure of the J1030 field that we obtained in 2017. This X-ray field has a nominal aim point centered on the quasar (QSO) SDSS J1030+0525 at $z=6.31$
(Fan et al. 2001). This QSO was one of the first $z \sim 6$ QSOs discovered by the Sloan Digital Sky Survey (SDSS), and has also been observed by the Hubble Space Telescope Advanced Camera for Surveys (HST/ACS; Stiavelli et al. 2005; Kim et al. 2009), by the HST Wide Field Camera 3 (HST/WFC3; PI Simcoe, unpublished), and by the Very Large Telescope Multi-Unit Spectroscopic Explorer (VLT/MUSE; ESO archive). Its field is part of the Multiwavelength Yale-Chile survey (MUSYC; Gawiser et al. 2006), which provides imaging in UBVRIzJHK down to $B=26$ and $K=23$ AB (Quadri et al. 2007), and has also been entirely observed by Spitzer IRAC down to $22.5 \mathrm{AB}$ mag at $4.5 \mu \mathrm{m}$ (Annunziatella et al. 2018). Near-infrared (NIR) spectroscopy (ISAAAC/VLT) showed that SDSS J1030+0524 is powered by a BH with a mass of $1.4 \times$ $10^{9} M_{\odot}$ (derived from the Mg II emission line; Kurk et al. 2007; De Rosa et al. 2011). Deep and wide optical and NIR imaging observations of the region $\left(\sim 25^{\prime} \times 25^{\prime}\right)$ around the QSO with LBT/LBC and CHFT/WIRCam (corresponding to a region of $8 \times 8 \mathrm{Mpc}$ at $z=6.31$ ) also showed that this field features the clearest evidence to date of an overdense region around a $z \sim 6$ QSO (Morselli et al. 2014; Balmaverde et al. 2017). The main goals of our deep Chandra observation of J1030 are the following: (i) to obtain one of the highest quality spectrums ever achieved in the X-rays for a QSO at $z \sim 6$ (see Nanni et al. 2018), and (ii) to perform a deep X-ray survey in a candidate highly biased region of the Early Universe that has excellent multi-band coverage. These data were used to study the X-ray variability of the $z=6.31$ QSO SDSS J1030+0524 (Nanni et al. 2018), as well as the diffuse emission detected southward of the QSO associated to a galaxy overdensity at $z=1.7$ (Nanni et al. 2018; Gilli et al. 2019), and to characterize the obscured AGN 
Table 1. SDSS J1030+0524 observation log.

\begin{tabular}{lccccc}
\hline \hline \multirow{2}{*}{ ObsID } & \multirow{2}{*}{ Date } & \multirow{2}{*}{$\theta^{(a)}$} & \multirow{2}{*}{$t_{\mathrm{exp}}{ }^{(b)}$} & \multicolumn{2}{c}{ Aim point } \\
\cline { 5 - 6 } & & {$\left[{ }^{\circ}\right]$} & {$[\mathrm{ks}]$} & $\alpha(\mathrm{J} 2000)$ & $\delta(\mathrm{J} 2000)$ \\
\hline 18185 & 2017 Jan. 17 & 64.2 & 46.3 & 103028.35 & +052540.2 \\
19987 & 2017 Jan. 18 & 64.2 & 126.4 & 103028.35 & +052535.3 \\
18186 & 2017 Jan. 25 & 64.2 & 34.6 & 103028.35 & +052535.3 \\
19994 & 2017 Jan. 27 & 64.2 & 32.7 & 103028.35 & +052537.6 \\
19995 & 2017 Jan. 27 & 64.2 & 26.7 & 103028.35 & +052534.2 \\
18187 & 2017 Mar. 22 & 259.2 & 40.4 & 103026.67 & +052407.1 \\
20045 & 2017 Mar. 24 & 259.2 & 61.3 & 103026.66 & +052407.5 \\
20046 & 2017 Mar. 26 & 259.2 & 36.6 & 103026.56 & +052413.3 \\
19926 & 2017 May 25 & 262.2 & 49.4 & 103026.68 & +052414.2 \\
20081 & 2017 May 27 & 262.2 & 24.9 & 103026.66 & +052415.2 \\
\hline
\end{tabular}

Notes. ${ }^{(a)}$ Roll angle in degrees of the ACIS-I instrument. ${ }^{(b)}$ Exposure time after background flare removal.

in the field (Peca et al., in prep.). In particular, the $z=1.7$ overdensity is composed of seven galaxy members (six of which are star forming) around a central Compton-thick FRII radio source, the eastern radio lobe of which is laying at the center of the diffuse X-ray emission and is likely promoting the star formation of the nearby overdensity galaxy members (Gilli et al. 2019). All these considerations make J1030 a legacy field for the study of large-scale structures around distant accreting SMBHs. Based on the multi-wavelength coverage of the field, here we present multi-wavelength identifications and basic multi-wavelength photometry for the detected X-ray sources and their optical/IR counterparts.

The paper is organized as follows. In Sect. 2 we describe the Chandra data, and the data reduction procedure. In Sect. 3 we report the X-ray source detection procedure with a detailed description of the analysis of source completeness and reliability. In Sect. 4, we present the main X-ray source catalog, and provide the X-ray sources characterization and multi-wavelength identifications. In Sect. 5, we present the cumulative number counts for the main source catalog, and in Sect. 6 we provide a summary of the main results. Throughout this paper we assume $H_{0}=70 \mathrm{~km} \mathrm{~s}^{-1} \mathrm{Mpc}^{-1}, \Omega_{\Lambda}=0.7$, and $\Omega_{\mathrm{M}}=0.3$ (Bennett et al. 2013 ), and errors are reported at $68 \%$ confidence level if not specified otherwise. Upper limits are reported at the $3 \sigma$ confidence level.

\section{Observations and data reduction}

The SDSS J1030+0524 field was observed by Chandra with ten different pointings between January and May 2017 for a total exposure of $\sim 479 \mathrm{ks}$. Observations were taken in the $v$ faint mode for the event telemetry format using the Advanced CCD Imaging Spectrometer (ACIS) instrument with a roll angle of $\sim 64^{\circ}$ for the first five observations and $\sim 259^{\circ}$ for the others. The ten observations (hereafter ObsIDs) cover a total area of roughly $335 \mathrm{arcmin}^{2}$ and the exposure times of the individual observations range from 26.7 to $126.4 \mathrm{ks}$. A summary of the observations is provided in Table 1.

The data were reprocessed using the Chandra software CIAO v. 4.8. Data analysis was carried out using only the events with ASCA grades 0, 2, 3, 4, and 6. We then produced X-ray images in the soft, hard, and full bands for each ObsID.

After the data reduction, we corrected the astrometry (applying shift and rotation corrections) of the individual ObsIDs using as reference the WIRCam catalog, which contains

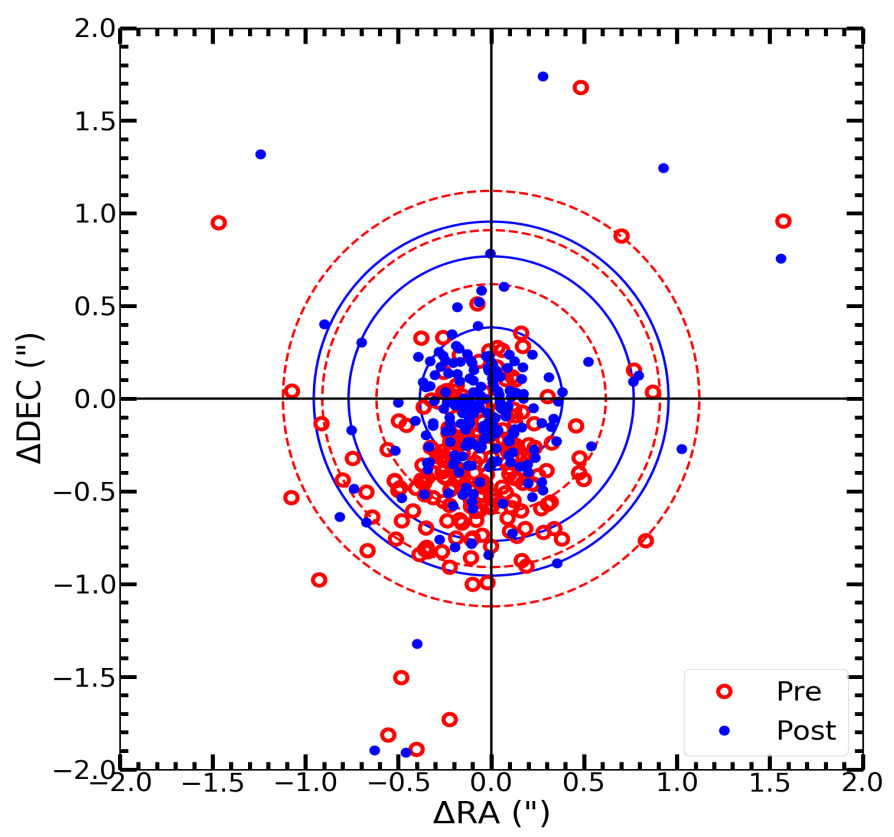

Fig. 2. X-ray to $J$-band separation $(\triangle \mathrm{RA}, \Delta \mathrm{Dec})$ in arcsec for $\mathrm{X}$-ray sources detected in each single observation (with a wavdetect falsepositive probability detection threshold set to $10^{-6}$ and off-axis $<6^{\prime}$; see Sect. 2 for the details) before (red open circles) and after (blue solid circles) astrometric correction. The circles encompass 68\%, 90\%, and $95 \%$ of the sources before (red dashed line) and after (blue solid line) astrometric correction.

$J$-band selected sources down to $J_{\mathrm{AB}}=23.75$ (Balmaverde et al. 2017). First, we created exposure maps and point spread function (PSF) maps for all ObsIDs using the CIAO tools fluximage and mkpsfmap, respectively. The exposure and PSF maps were computed for $90 \%$ of the encircled energy fraction (EEF) and at an energy of 2.3,1.4, and $3.8 \mathrm{keV}$ for the full, soft, and hard bands, respectively. We then ran the Chandra source detection task wavdetect (Freeman et al. 2002) on the $0.5-7 \mathrm{keV}$ images to detect sources to be matched with the $J$-band detected objects. We set the false-positive probability detection threshold to a conservative value of $10^{-6}$ and used a " $\sqrt{2}$ sequence" of wavelet scales up to 8 pixels (i.e., 1.41, 2, 2.83, 4, 5.66, and 8 pixels) in order to detect only the brightest sources with a well-defined X-ray centroid. For the match we considered only 43 X-ray sources with a positional error ${ }^{1}$ below $\sim 0.4^{\prime \prime}$ and offaxis $<6^{\prime}$. We used the CIAO tool wcs_match and wcs_update to match these 43 sources and correct the astrometry, and create new aspect solution files. We considered a matching radius of $2^{\prime \prime}$ and applied both translation and rotation corrections. The new aspect solutions were then applied to the event files and the detection algorithm was run again (using the same wavdetect parameters and criteria previously adopted). The applied astrometric correction reduces the mean angular distance between the $\mathrm{X}$-ray sources and their $J$-band counterparts from $\theta=0.253^{\prime \prime}$ to $\theta=0.064^{\prime \prime}$. As shown in Fig. 2, we found that, after applying the astrometric corrections, the distance $(d)$ between the $\mathrm{X}$-ray sources used for the astrometric correction and the optical counterparts is $d<0.38^{\prime \prime}, 0.77^{\prime \prime}, 0.95^{\prime \prime}$ for $68 \%, 90 \%$, and $95 \%$ of the X-ray sources, respectively (to be compared with

\footnotetext{
1 Computed as: $\sqrt{\sigma_{\mathrm{RA}}^{2}+\sigma_{\mathrm{Dec}}^{2}}$, where $\sigma_{\mathrm{RA}}$ and $\sigma_{\mathrm{Dec}}$ are the errors on
} right ascension and declination, respectively, from wavdetect. 


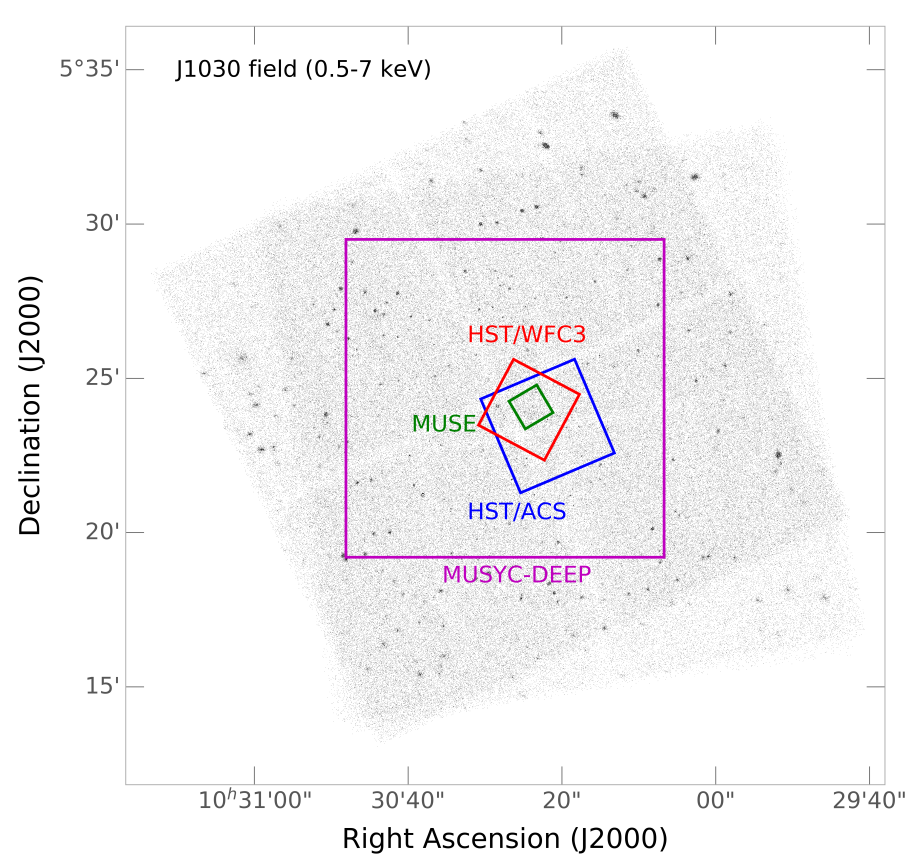

Fig. 3. Full-band $(0.5-7 \mathrm{keV})$ Chandra ACIS-I image of the SDSS J1030+0524 field in logarithmic gray scale. The multi-color regions show some of the central multi-wavelength coverage of the field: the MUSYC-DEEP in purple, HST/WFC3 in red, HST/ACS in blue, and VLT/MUSE in green.

$d<0.62^{\prime \prime}, 0.91^{\prime \prime}, 1.12^{\prime \prime}$ before the correction). Despite the astrometric corrections, a mean offset of $\triangle \mathrm{RA}=-0.07 \pm 0.3$ and $\Delta \mathrm{Dec}=-0.1 \pm 0.4$ is still present. We performed several tests, changing both the off-axis angles and the full band net counts cuts, to verify whether or not the offset is due to a particular source (or a group of them), but the offset persists and is consistent with the values reported. However, this offset is unlikely to affect the matching analysis described in Sect. 4.2, as the X-ray sources positional errors are generally larger.

Finally, we stacked the corrected event files using the reproject_obs task and created X-ray images from the merged event file using the standard ASCA grade set in the full, soft, and hard bands. In Fig. 3 we display the final Chandra full-band image with the coverage of the innermost multi-wavelength fields mentioned in Sect. 1. A false-color X-ray image of the field is shown in Fig. 4. The individual PSF maps were combined using the task dmimgcalc to return the exposure-weighted average PSF value at each pixel location in the combined mosaic, while the individual effective-exposure maps were summed together to obtain the total effective-exposure map of the field in the full, soft, and hard bands. The full-band effective-exposure map is shown in Fig. 5.

\section{X-ray source detection}

The X-ray source detection procedure follows a two-stage approach that has also been adopted in past deep X-ray surveys such as the CDF-S (i.e., Xue et al. 2011; Luo et al. 2017), and the CDF-N (Xue et al. 2016): a preliminary list of source candidates was initially generated by wavdetect source detection and was then filtered after photometry performed with ACIS Extract (AE; Broos et al. 2012) to produce our final source catalog.

\subsection{Generation of the preliminary catalog}

To generate the preliminary candidate source list, we ran wavdetect on the merged images in the full, soft, and hard bands using a $\sqrt{2}$ sequence of wavelet scales up to 16 pixels (i.e., $1.414,2$, $2.828,4,5.656,8,11.314$, and 16 pixels) and a false-positive probability threshold of $10^{-4}$. We also provided wavdetect with the average PSF maps for each energy band. This produced 498, 383, 370 candidate sources in the full, soft, and hard bands, respectively. Among them, 289, 221, and 218 sources are also detected in the full, soft, and hard bands, respectively, when running wavdetect with a more conservative threshold of $10^{-5}$ that is used in many deep Chandra surveys. The loose wavdetect source-detection threshold of $10^{-4}$ is expected to introduce a large number of spurious detections that must be filtered out, but also allows us to push the detection to the faintest possible limits.

We then improved the source positions through the AE "CHECK_POSITIONS" procedure and used AE to extract photometric properties of the candidate sources. The details of the AE photometric extraction are described in the AE User's Guide, and a summary is also provided in Xue et al. (2011). We used AE to perform source and background extractions for each source in each ObsID and then we merged the results. In our case, a polygonal extraction region that approximates the $\sim 90 \%$ encircled energy fraction contour of the local PSF, at $E=1.4 \mathrm{keV}$ in the full and soft bands, and at $E=2.3 \mathrm{keV}$ in the hard band, was utilized to extract source counts. We adopted the AE "BETTER_BACKGROUNDS" algorithm for background extraction (see Sect. 7.6.1 of the AE User's Guide), in order to obtain a single background region plus a background scaling that simultaneously models all background components, including the background that arises from the PSF wings of neighboring sources. A minimum number of 100 counts in the merged background spectrum is required to ensure photometric accuracy, which was achieved through the AE "ADJUST_BACKSCAL" stage. The extraction results from individual observations were then merged to produce photometry for each source through the AE "MERGE_OBSERVATIONS" stage. To filter the preliminary catalog, the most important output parameter from AE is the binomial no-source probability $\left(P_{\mathrm{B}}\right)$, which is the probability of observing at least the same number of source counts under the assumption that there is no real source at that location and that the observed number of counts is purely due to a background fluctuation:

$P_{\mathrm{B}}(X \geq S)=\sum_{X=S}^{N} \frac{N !}{X !(N-X) !} p^{X}(1-p)^{N-X}$,

where $S$ is the total number of counts in the source-extraction region (before background subtraction); $N=S+B_{\text {ext }}$, where $B_{\text {ext }}$ is the total number of counts in the background extraction region; and $p=1 /(1+$ BACKSCAL $)$ with BACKSCAL $=A_{\text {ext }} / A_{\text {src }}$ is the ratio between the background and source extraction regions. We computed $P_{\mathrm{B}}$ for each source in all three (full, soft, hard) bands. Although $P_{\mathrm{B}}$ is a classic confidence level, it is usually not a good indicator of the fraction of spurious sources (e.g., a cut at $P_{\mathrm{B}}=0.01$ does not correspond to a $1 \%$ spurious rate), mainly because the extractions were performed on a biased sample of candidate sources that already survived a filtering process by wavdetect. Furthermore, given its definition, the value of $P_{\mathrm{B}}$ is dependent on the choice of source and background extraction regions. Therefore, we cannot reject spurious sources simply based on the absolute value of $P_{\mathrm{B}}$ itself. A $P_{\mathrm{B}}$ threshold derived from simulations needs to be adopted to maximize the completeness and reliability of our sample. 


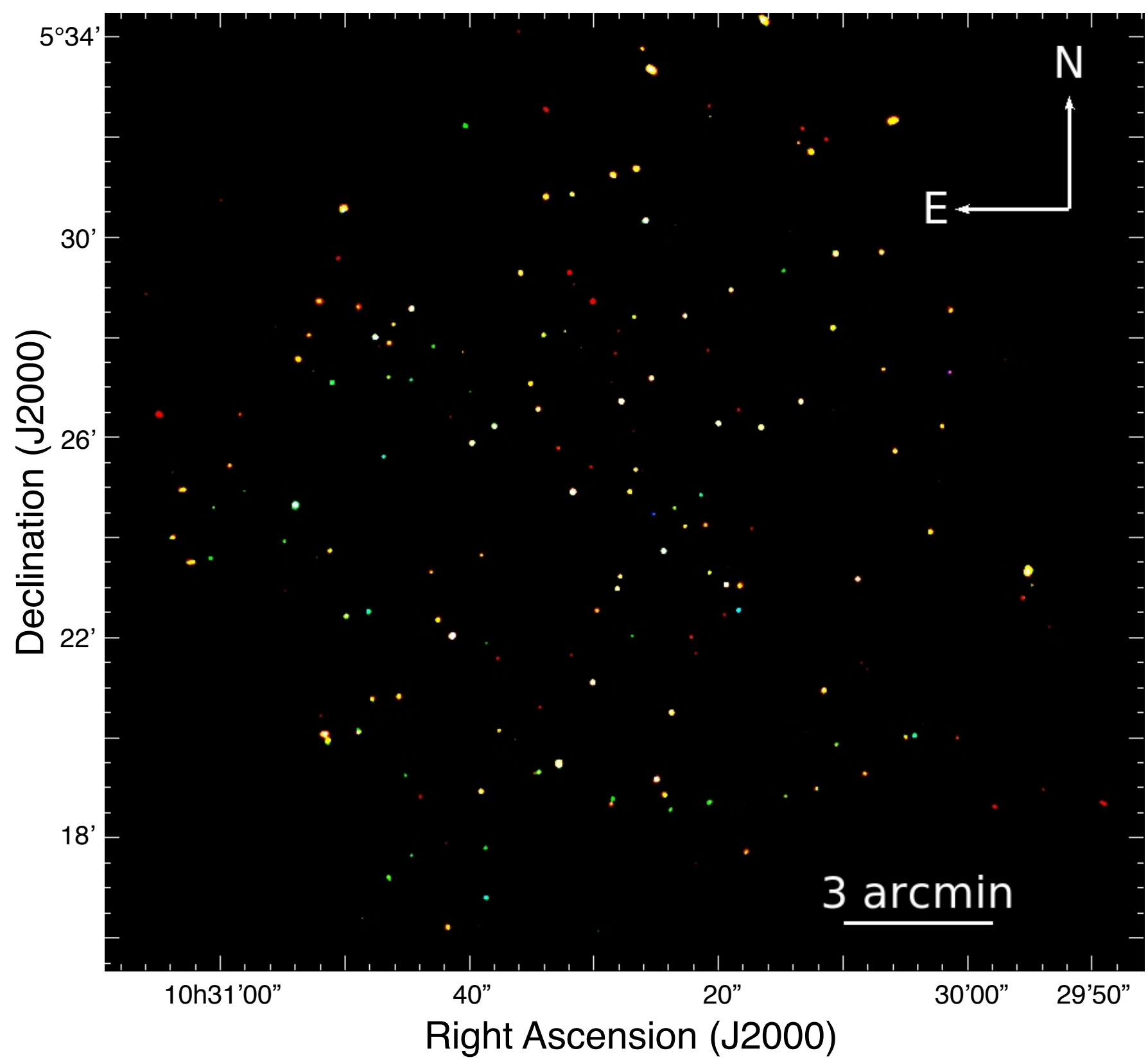

Fig. 4. Smoothed "false-color" image of the SDSS J1030+0524 field. Colors correspond to $0.5-2.0 \mathrm{keV}$ (red), 2-4.5 keV (green), and 4.5-7 keV (blue).

\subsection{Generation of the simulated data}

To clean the catalog of spurious sources as much as possible and to assess the completeness and reliability of our final sample we produced three simulations that closely mirror our observations. A similar procedure was used in previous X-ray surveys (e.g., Cappelluti et al. 2007, 2009; Puccetti et al. 2009; Xue et al. 2011, 2016; Luo et al. 2017).

First, we considered a mock catalog of X-ray sources (AGNs and normal galaxies) that covers an area of one square degree and reaches fluxes that are well below the detection limit of our $\sim 479 \mathrm{ks}$ exposure. In this mock catalog, we assigned to each simulated AGN a soft-band flux randomly drawn from the soft-band $\log (N)-\log (S)$ relation expected in the AGN population synthesis model by Gilli et al. (2007). Simulated galaxy fluxes were drawn randomly from the soft-band galaxy $\log (N)-$ $\log (S)$ relation of the "peak-M" model of Ranalli et al. (2005).
The AGN and galaxy integrated flux is consistent within the uncertainties with the cosmic X-ray background flux (CXB; see e.g., Cappelluti et al. 2017). Active galactic nuclei and galaxies were simulated down to $2 \times 10^{-18} \mathrm{erg} \mathrm{cm}^{-2} \mathrm{~s}^{-1}$ in the $0.5-2 \mathrm{keV}$ band in order to include the contribution of undetectable sources that produce the spatially nonuniform background component. The soft-band fluxes of the simulated AGNs and galaxies were converted into full-band fluxes assuming power-law spectra with $\Gamma=1.4^{2}$ and $\Gamma=2.0$, respectively. The number of simulated sources has been rescaled for the J1030 field area $\left(\sim 335 \operatorname{arcmin}^{2}\right)$, and source coordinates were randomly assigned within that area. We used the MARX

2 This value is typically used to translate count rates into fluxes for the AGN population, since it describes fairly well the observed slope of the CXB and is therefore representative of a sample that includes both unobscured and obscured AGNs (Hickox \& Markevitch 2006). 


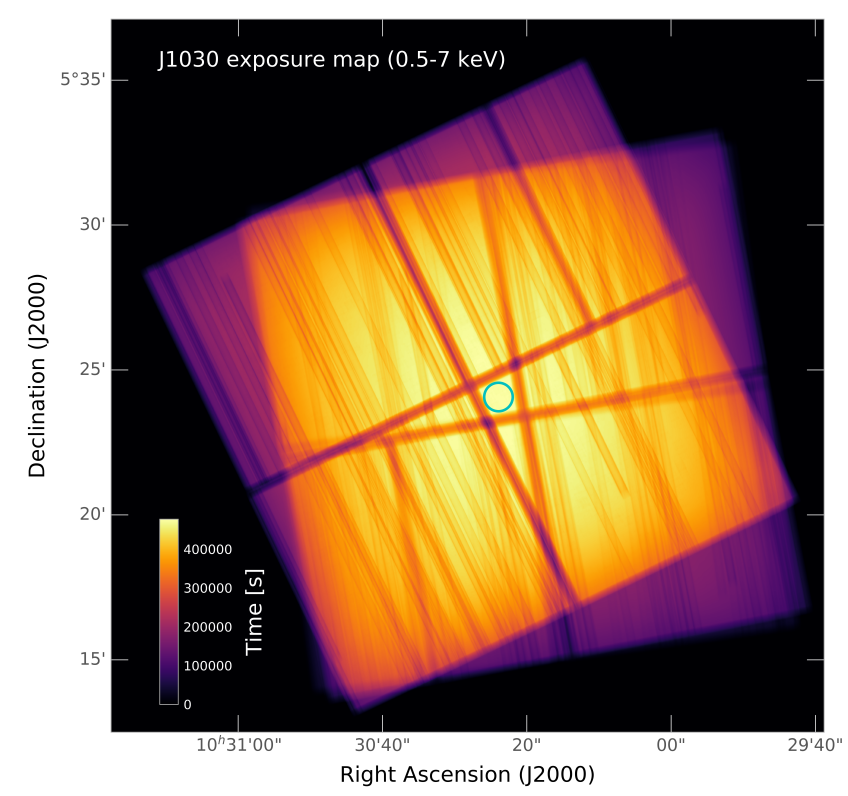

Fig. 5. Full-band $(0.5-7 \mathrm{keV})$ Chandra effective-exposure map of the SDSS J1030+0524 field. The linear scale color bar is shown in the bottom left; displayed effective exposure times are in units of s. The cyan circle $\left(r=30^{\prime \prime}\right)$ marks the position of the $z=6.31$ QSO SDSS J1030+0524.

software (v. 5.3.3; Davis et al. 2012) to convert source fluxes to a Poisson stream of dithered photons and to simulate their detection by ACIS-I.

Second, we produced ten simulated ACIS-I observations of the mock catalog, each configured to have the same aim point, roll angle, exposure time, and aspect solution file of each of the ten J1030 pointings (see Table 1). These simulated source event files contain the actual number of photons produced on the detector by our simulated sources. We then produced the corresponding background event files from the J1030 event files. For each real event file, we masked all the events associated to our preliminary source candidates and then filled the masked regions with events that obey the local probability distribution of background events (using the blanksky and blanksky_sample tools). The simulated source event files produced using MARX were then merged with the background event files to produce ten simulated ACIS-I pointings that closely mirror the ten real ones. above:

As a final step, we followed the same approach adopted

- We stacked the ten simulated event files using the reproject_obs task and created X-ray images in all the three X-ray bands from the merged event file (Sect. 2).

- We ran wavdetect on each simulated combined image at a false-positive probability threshold of $10^{-4}$ to produce a catalog of simulated source candidates, and used AE to perform photometry (including the $P_{\mathrm{B}}$ values) on them (Sect. 3.1).

The procedure above was repeated three times, allowing us to generate a total of three complete simulations that mirror the J1030 field and to obtain a simulated preliminary source catalog from each simulation.

\subsection{Completeness and reliability}

Simulations are the best tools to set a probability threshold $\left(P_{\mathrm{B}}\right)$ for source filtering because we have full control of the input and output sources. After creating the three simulations and the corresponding candidate source catalogs (as described in Sect. 3.2), we matched the detected output simulated sources with the input sources in the mock catalog using a likelihood-ratio matching technique (see e.g., Ciliegi et al. 2003; Brusa et al. 2007). The goal of this method is to distinguish between input simulated sources and spurious sources among those detected in the simulation. Once the detected sources were classified, we adopted other filters based on the X-ray properties of each source (like the $P_{\mathrm{B}}$; see the detailed description reported below) that can also be applied to our Chandra observation.

Briefly, our likelihood-ratio (LR) technique takes into account the positional accuracy of the output sources, and also the expected flux distribution of the input ones. It assigns likelihood and reliability parameters to all possible counterparts, and mitigates the effect of false matches. For an input source with a flux $f$ at an angular separation $r$ from a given output source, the LR is the ratio between the probability of the input being the true counterpart of the output and the corresponding probability of the input being an unrelated (i.e., background) object (e.g., Sutherland \& Saunders 1992; Brusa et al. 2005):

$\mathrm{LR}=\frac{F(r) q(f)}{n(f)}$

where $F(r)$ is the probability distribution function of the angular separation, $q(f)$ is the expected flux distribution of the input sources, and $n(f)$ is the surface density of background objects with flux $f$. We refer to Appendix A for a complete explanation of Eq. (2). In our case, for each output source we searched for input sources inside a circular area of $r_{\mathrm{LR}}=5^{\prime \prime}$ (following Luo et al. 2010) centered on the output position to allow matching of the X-ray sources at large off-axis angles.

A threshold for $\mathrm{LR}\left(\mathrm{LR}_{\mathrm{th}}\right)$ is needed to discriminate between spurious and real associations: an output source is considered to have an input counterpart if its $L R$ value exceeds $L_{\text {th }}$. The choice of $L R_{t h}$ depends on two factors: first, $L R_{t h}$ should be small enough to avoid missing many real identifications, so that the sample completeness is high; second, $\mathrm{LR}_{\mathrm{th}}$ should be large enough to keep the number of spurious identifications low in order to increase the reliability of the identifications. The reliability of a single input source $j$, which represents the probability of being the correct identification, is defined as:

$R_{j}=\frac{\mathrm{LR}_{j}}{\sum_{i} \mathrm{LR}_{i}+(1-Q)}$,

where the sum is over all the possible input counterparts for an output source $i$, and $Q=\int_{f_{\mathrm{lim}}}^{+\infty} q(f) \mathrm{d} f$ is the probability that the input counterpart $j$ is brighter than the flux limit $\left(f_{\lim }\right)$ of the catalog. We then defined the reliability parameter $(R)$ for the total sample as the ratio between the sum of the reliabilities of all sources identified as possible counterparts and the total number of sources with $\mathrm{LR}>\operatorname{LR}_{\mathrm{th}}\left(N_{\mathrm{LR}>\mathrm{LR}_{\mathrm{th}}}\right)$ :

$R=\frac{\left(\sum_{j} R_{j}\right)_{\mathrm{LR}>\mathrm{LR}_{\mathrm{th}}}}{N_{\mathrm{LR}>\mathrm{LR}_{\mathrm{th}}}}$

We also measure the completeness parameter $(C)$ of the total sample defined as the ratio between the sum of the reliability of all sources identified as possible counterparts and the total number of output sources $\left(N_{\mathrm{X}}\right)$ :

$C=\frac{\left(\sum_{j} R_{j}\right)_{\mathrm{LR}>\mathrm{LR}_{\mathrm{th}}}}{N_{\mathrm{X}}}$. 
As proposed in Brusa et al. (2007) and Civano et al. (2012), $\mathrm{LR}_{\mathrm{th}}$ was computed as the likelihood-ratio that maximizes the quantity $(R+C) / 2$ (we found $\mathrm{LR}_{\mathrm{th}}=3.61,4.05,3.31$ for the full, soft, and hard band, respectively). We hence flagged those sources with $\mathrm{LR}>\mathrm{LR}_{\text {th }}$ as good matches and those with $\mathrm{LR}<\mathrm{LR}_{\text {th }}$ as spurious matches.

We used those matches flagged as "good" to assess the completeness and reliability of the simulated catalog. Looking at the distributions of the net counts in the three bands (full, soft, and hard) versus off-axis angle for both good and spurious matches, we derived three empirical linear cut relations (one for each band) that define an effective source-count limit as a function of the off-axis angles. These cut lines (blue lines in Fig. 6) maximize the number of rejected spurious sources while keeping the number of rejected "good" sources around 10\%. By considering only those sources above the cut lines, we then defined the completeness as the ratio between the number of "good" sources detected with a binomial probability above a certain value and the total number of "good" sources. The reliability is defined as 1 minus the ratio between the number of spurious sources above a certain probability value and the total number of sources.

In Fig. 7 we show the completeness (black solid line) and reliability (red dashed line) as a function of the $P_{\mathrm{B}}$ for the simulations in the full, soft, and hard bands for sources that survived our count-limit relation cuts. We adopted a probability threshold value of $P_{\mathrm{B}}=2 \times 10^{-4}$ to keep the reliability values $>95 \%$ in all the three bands. In fact, adopting $P_{\mathrm{B}}=2 \times 10^{-4}$, the completeness levels for the entire $\mathrm{J} 1030$ field are $95 \%$ (full band), $97 \%$ (soft band), and 91\% (hard band), while the reliability levels are 95\% (full band), 96\% (soft band), and 95\% (hard band). Finally, we applied our source-count limit cut and the $P_{\mathrm{B}}=2 \times 10^{-4}$ threshold derived from the simulations as filters for our J1030 preliminary real source catalog: 244, 193, and 208 sources survived in the full, soft, and hard bands, respectively. Compared to past X-ray surveys, this is the first time that more sources are detected in the hard band rather than in the soft one, and we ascribed this difference to the rapid degradation of the soft-band Chandra effective area that occurred in the last few years.

We then matched the three band catalogs using a matching radius $r=5^{\prime \prime}$ (for $5^{\prime \prime}<r<10^{\prime \prime}$ no additional sources are matched) and visually checked all matches. The final catalog contains 256 unique sources detected in at least one band.

We also computed the completeness and reliability (following the same approach described above) for source catalogs generated with a wavdetect false-positive probability threshold of $10^{-5}$. Based on simulations, we verified that catalogs generated with wavdetect threshold $10^{-5}$ and $\left(P_{\mathrm{B}}=10^{-3}\right)$ have the same reliability and completeness and similar source numbers to those obtained using $10^{-4}$ and $P_{\mathrm{B}}=2 \times 10^{-4}$. After a visual check of those sources that are not in common between the two catalogs, we found that the catalog obtained with a wavdetect threshold of $10^{-4}$ and $P_{\mathrm{B}}=2 \times 10^{-4}$ contains more associations with optical/IR counterparts than the other one, and we hence adopt it as our final X-ray source catalog.

\section{Source catalog}

As explained in Sect. 3.3, our final catalog consists of 256 sources detected in one or more X-ray bands (full, soft, and hard); in Table 2 we report the total number of sources for each band combination.

Eleven sources are detected only in the full band, six only in the soft band, and six only in the hard band. For each source
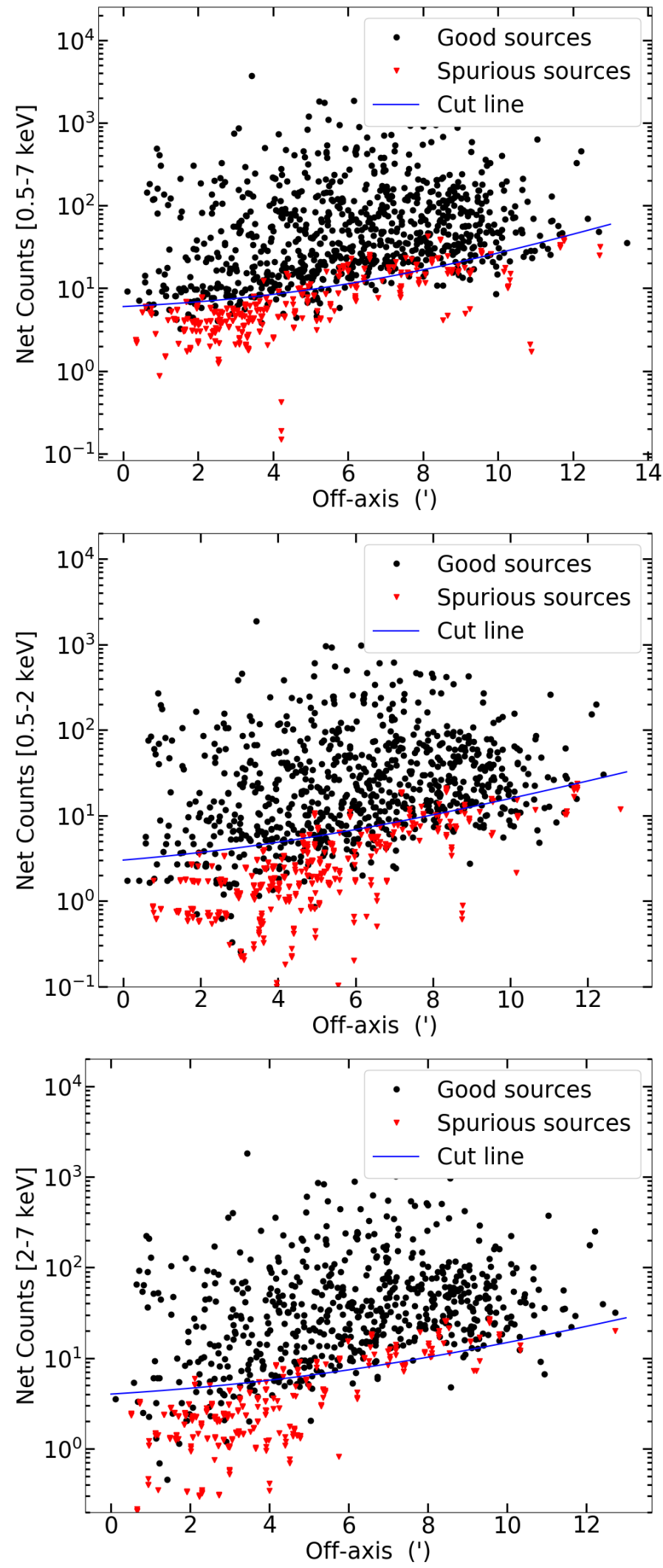

Fig. 6. Net counts vs. off-axis angles for the sources detected in the three simulations in the full (top), soft (middle), and hard (bottom) bands. The black dots are the "good" input-output matches according to the likelihood-ratio method, while the red triangles are those considered as spurious. The blue line represents the source-count limit as a function of the off-axis angle adopted to compute the completeness and reliability. 

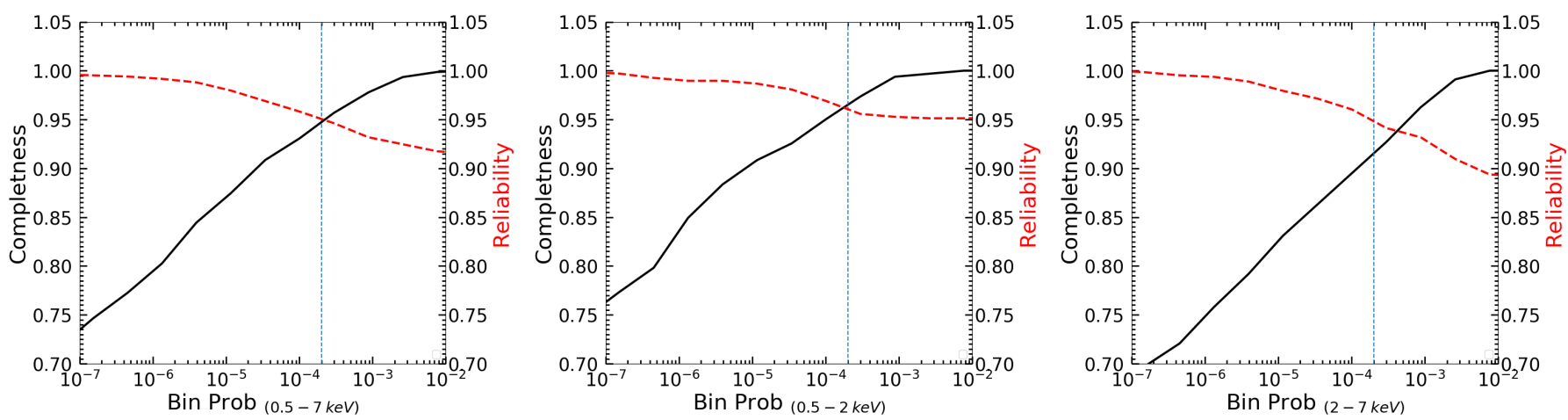

Fig. 7. Completeness $(C$, black curve) and reliability ( $R$, red dashed curve) as a function of the binomial no-source probability for the three combined simulations in the full (left), soft (middle), and hard (right) bands, respectively. The dashed vertical line indicates the chosen sourcedetection threshold of $P_{\mathrm{B}}=2 \times 10^{-4}$.

Table 2. Number of Chandra sources detected in up to three bands.

\begin{tabular}{lc}
\hline \hline $\begin{array}{l}\text { Band } \\
{[\mathrm{keV}]}\end{array}$ & $\begin{array}{c}\text { Number of } \\
\text { sources }\end{array}$ \\
\hline$F+S+H$ & 156 \\
$F+S$ & 31 \\
$F+H$ & 46 \\
$F$ & 11 \\
$S$ & 6 \\
$H$ & 6 \\
Total & 256 \\
\hline
\end{tabular}

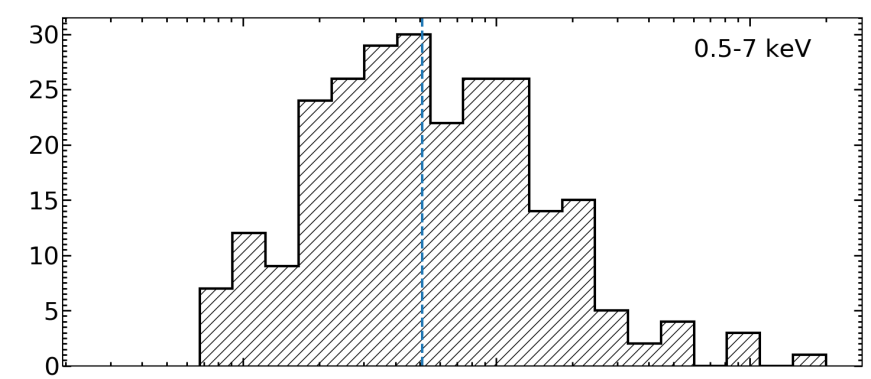

Notes. The bands reported are the full $(F)$, soft $(S)$, and hard $(H)$.

Table 3. Statistics of Chandra detected sources.

\begin{tabular}{lccccc}
\hline \hline \multirow{2}{*}{$\begin{array}{l}\text { Band } \\
{[\mathrm{keV}]}\end{array}$} & Number of & \multicolumn{4}{c}{ Net counts per source } \\
\cline { 3 - 6 } & sources & Max & Min & Mean & Median \\
\hline Full & 244 & 1849.3 & 6.7 & 96.5 & 50.9 \\
Soft & 193 & 1196.1 & 2.7 & 61.0 & 41.5 \\
Hard & 208 & 647.6 & 3.4 & 53.3 & 40.7 \\
\hline
\end{tabular}

we derived the net counts, hardness ratio (HR), and band fluxes and relative errors or upper limits (for those sources that are not detected in a given band), as described in Sect. 4.1. In Table 3 we report the basic statistics of the source counts in the three bands.

\subsection{X-ray properties}

In each of the three X-ray bands, the net source counts were derived from the AE "MERGE_OBSERVATIONS" procedure using a polygonal extraction region that approximates the $\sim 90 \%$ of the encircled energy fraction at $E=1.4 \mathrm{keV}$ in the full and soft bands, and at $E=3.8$ in the hard band, as explained in Sect. 3, while the associated $1 \sigma$ errors are computed by $\mathrm{AE}$ following Gehrels (1986). For those sources that are below the detection threshold $\left(P_{\mathrm{B}}>2 \times 10^{-4}\right)$ in one or two bands, we computed the $3 \sigma$ upper limits using the srcflux tool of CIAO, which extracts source counts from a circular region centered at the source position that contains $90 \%$ of the PSF at $2.3,1.4$, and $3.8 \mathrm{keV}$ in the full, soft, and hard bands, respectively. Their counts from the background are extracted from an annular region around the source location that has an inner radius equal to the
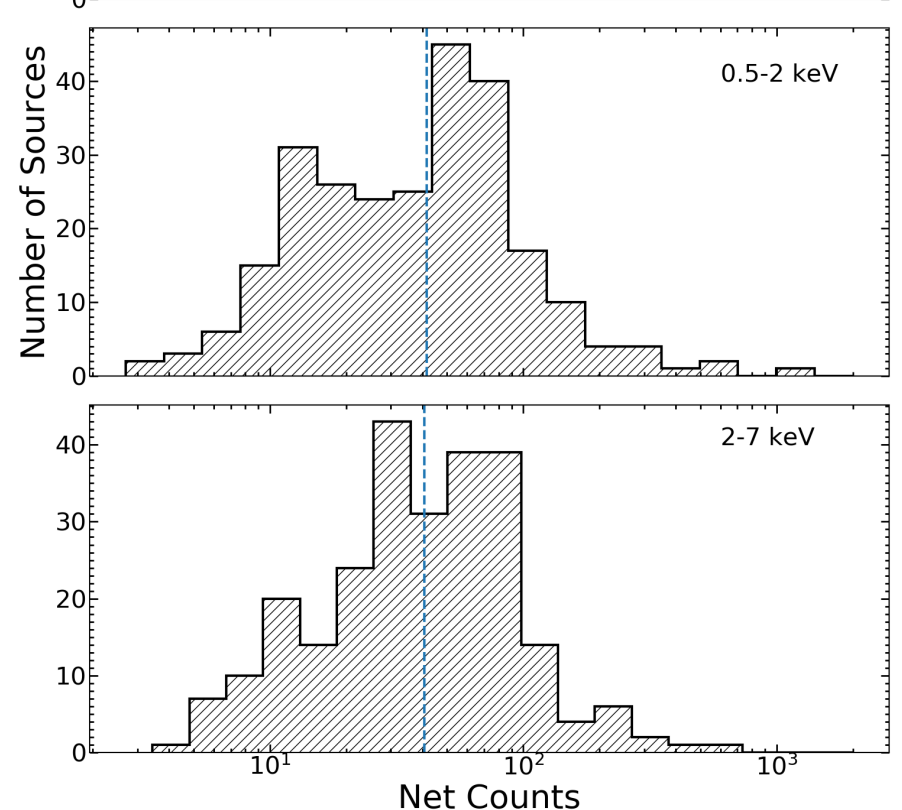

Fig. 8. Net counts distributions for the Chandra sources detected in the full (top), soft (middle), and hard (bottom) bands. The cyan dotted vertical lines mark the medians of the distributions: 50.9, 41.5, and 40.7 net counts for the full, soft, and hard bands, respectively. Sources with upper limits on the counts are not included in these plots.

size of the source radius and an outer radius five times larger. The distributions of the source counts in the three bands are displayed in Fig. 8.

We used the net count rates in the different bands to compute the HR for each source. The HR was computed as:

$\mathrm{HR}=\frac{H-S}{H+S}$ 
where $H$ and $S$ are the net rates (the ratio between the net counts and the effective exposure time at the source position) in the hard and soft bands, respectively. Errors are computed at the $1 \sigma$ level following the method described in Lyons (1991). Upper and lower limits were computed using the $3 \sigma$ net counts upper limits. For the 11 sources with only full-band detection, we could not compute the HR.

While a detailed spectral analysis of the X-ray sources is beyond the scope of the current study, we converted the aperture corrected count rates (or their upper limits) to the corresponding fluxes (or flux upper limits) in a given band assuming that their spectra are power-laws modified by only Galactic absorption $\left(N_{\mathrm{H}}=2.6 \times 10^{20} \mathrm{~cm}^{-2}\right)$ with effective power-law photon indices derived from the HRs. At fixed $N_{\mathrm{H}}$ and redshift, the HR is a function of the power-law index (e.g., see Fig. 10 in Marchesi et al. 2016). The $\mathrm{HR}-\Gamma$ relation was derived simulating $100 \mathrm{X}$-ray spectra with $N_{\mathrm{H}}=0, z=0$, and different $\Gamma=-2-+2$ (steps of 0.1 ), and deriving the corresponding HRs. For the sources not detected in both the soft and hard bands, the HRs cannot be constrained, and so we assumed a spectral power law with $\Gamma=1.4$ (i.e., the mean value derived for the slope of the CXB; Hickox \& Markevitch 2006) modified by Galactic absorption. In this case, the adopted count rate-to-flux conversion factors are $\mathrm{CF}=\mathrm{CR} /$ flux $=6.3,9.8,4.4 \times 10^{10}$ counts $\mathrm{erg}^{-1} \mathrm{~cm}^{2}$ for the full, soft, and hard bands, respectively. The distributions of the source fluxes in the three bands are displayed in Fig. 9.

\subsection{Multi-wavelength source identifications}

We searched for optical and IR counterparts of the X-ray sources in our LBT/LBC, CHFT/WIRCam, and Spitzer IRAC $(4.5 \mu \mathrm{m}$ band) catalogs (see Morselli et al. 2014; Balmaverde et al. 2017; Annunziatella et al. 2018, respectively) using a likelihood-ratio matching technique similar to that described in Sect. 3.3. Again, a threshold value for the likelihood-ratio that maximizes the $(R+C) / 2$ value was chosen $\left(\mathrm{LR}_{\mathrm{th}}=1.06,1.71,2.41,1.11\right.$ for the $r, z, J$, and IRAC bands, respectively). For the X-ray sources with multiple counterpart candidates that satisfy our likelihood threshold, we selected the candidate with the highest reliability level. In particular, we found 17,7 , and 9 X-ray sources that have multiple counterpart candidates that satisfy the likelihood threshold in the $r, z$, and $J$ bands, respectively, while there are no multiple counterpart candidates in the IRAC $4.5 \mu \mathrm{m}$ band.

For the optical and IR identifications, we used the following four catalogs:

- The J1030+0524 LBC $z$ and $r$ bands catalogs, which contain 29150 and 86150 sources with limiting AB magnitudes of 25.2 and 27.5, respectively (50\% completeness limit; Morselli et al. 2014). In Morselli et al. (2014) the $z$-band data were used as master images on which object detection $(5 \sigma)$ was made, and then the measurements were performed on the $r$-band images only to obtain spatially coherent photometric colors. Subsequently, we performed a source detection on the deeper $r$ image to produce an independent $r$-band catalog with a limiting $\mathrm{AB}$ magnitude of 27.5.

- The J1030+0524 WIRCam J-band (NIR) catalog that contains 14770 sources down to $J_{\mathrm{AB}}=23.75$ (50\% completeness limit at $5 \sigma$; Balmaverde et al. 2017).

- The J1030+0524 Spitzer IRAC at MIR $4.5 \mu \mathrm{m}$ band (MIR) catalog that contains 16317 sources down to $m_{4.5 \mu \mathrm{m}}=22.5(50 \%$ completeness limit at 5 $\sigma$; Annunziatella et al. 2018).

We initially identified unique counterparts for $244(95.3 \%)$ of the 256 main-catalog sources. We examined the $12 \mathrm{X}$-ray sources that lack counterparts and assigned multi-wavelength
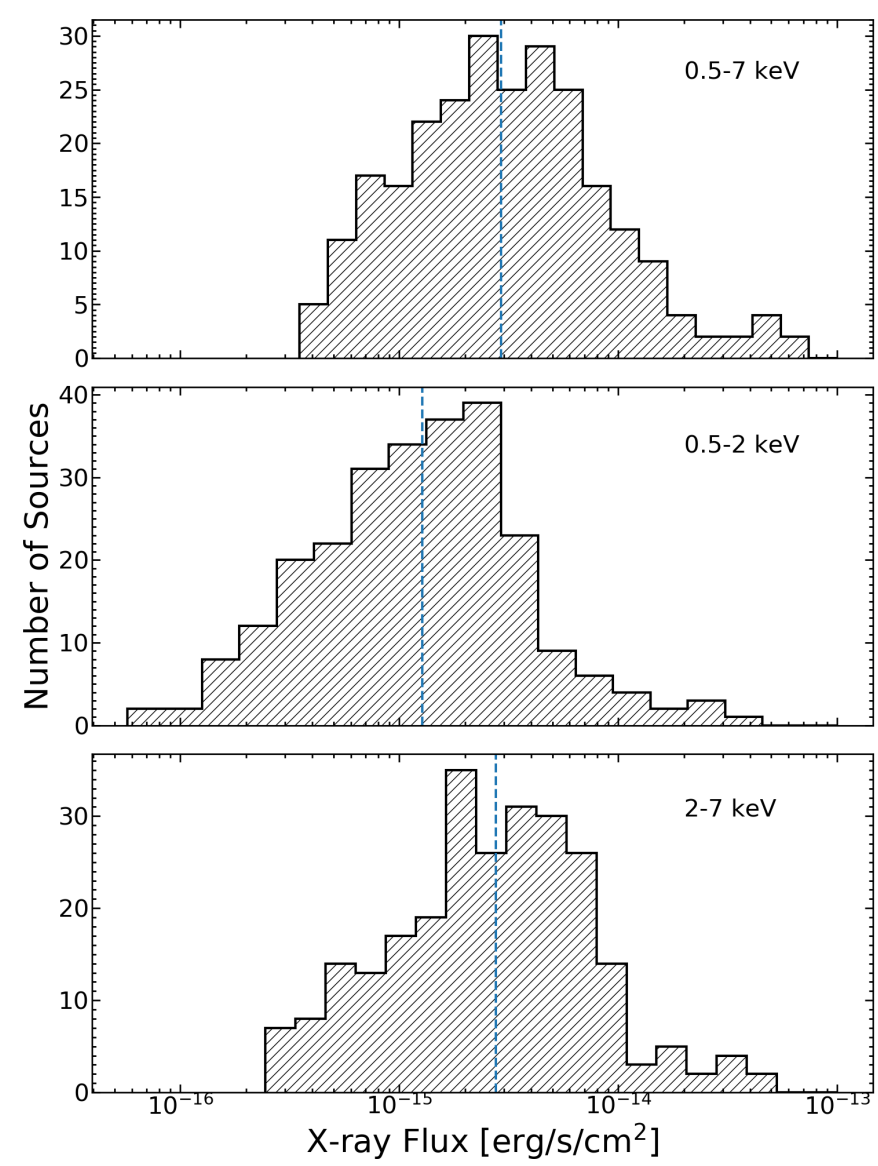

Fig. 9. Aperture-corrected X-ray flux distributions for the sources detected in the full (top), soft (middle), and hard (bottom) bands. The cyan dotted vertical lines mark the medians of the distributions: 2.9, 1.3, $2.8 \times 10^{-15} \mathrm{erg} \mathrm{s}^{-1} \mathrm{~cm}^{-2}$ for the full, soft, and hard bands, respectively. Sources with upper limits on the counts are not included in these plots.

matches to eight sources (with off-axis angle $>1^{\prime}$ and LR below but close to $\mathrm{LR}_{\mathrm{th}}$ ) for which the X-ray centroid computed by $\mathrm{AE}$ is too far away $\left(>1^{\prime \prime}\right)$ from the most likely optical counterpart to provide a LR value above our adopted threshold, while the positional error is consistent with the optical counterpart. After this adjustment, we then obtained primary counterparts in at least one optical/NIR/MIR band for $252(98.4 \%)$ of the 256 maincatalog sources. Among these 252 X-ray sources there are 1, 6 , and 6 sources that have $r, z$, or $J$ band counterparts, respectively, that are below the limiting magnitude of the corresponding survey. For these sources, we performed aperture photometry to obtain the missing catalog band magnitudes but we did not compute the corresponding likelihood and reliability because we have no information on the magnitude distribution of background sources at such faint fluxes. Postage-stamp images for the X-ray catalog sources are reported in Fig. A.2. The images are color composites of the LBT/LBC $r, z$, and CFHT/WIRCam $J$ bands. Furthermore, we show $17 \mathrm{X}$-ray sources in Fig. A.3 that only have IRAC counterparts. The distributions of the X-ray full-band fluxes versus LBT/LBC $r$-band, CHFT/ WIRCam $J$-band, and IRAC CH2 $4.5 \mu \mathrm{m}$-band magnitudes for the main catalog sources are displayed in Fig. 10. The blue diagonal lines show constant X-ray to $r$ - or $J$-band flux ratios defined (similarly to Civano et al. 2012) as:

$\log \left(f_{\mathrm{X}} / f_{\text {opt }}\right)=\log \left(f_{\mathrm{X}}\right)+C_{\text {opt }}+m_{\text {opt }} / 2.5=-1,0,+1$, 
where $f_{\mathrm{X}}$ is the X-ray full-band flux, $m_{\mathrm{opt}}$ is the magnitude at the chosen optical/IR band, and $C_{\text {opt }}$ is a constant which depends on the specific filter used in the optical observations. Considering the bandwidths and the effective wavelength of the LBC $r$-band, WIRCam $J$-band, and IRAC CH2 $4.5 \mu \mathrm{m}$-band filters, we used $C_{r}=5.41, C_{J}=5.96$, and $C_{4.5 \mu \mathrm{m}}=6.27$. The yellow shaded region between the blue diagonal dashed lines $(-1<$ $\left.\log \left(f_{\mathrm{X}} / f_{\text {opt }}\right)<+1\right)$ in Fig. 10 has been adopted as the reference area where unobscured AGNs are expected to lay in the optical bands, while obscured AGNs are expected at $\log \left(f_{\mathrm{X}} / f_{\text {opt }}\right)>1$ (e.g., Brusa et al. 2010; Civano et al. 2012). The higher number of sources above the $\log \left(f_{\mathrm{X}} / f_{\text {opt }}\right)=1$ relation observed in the $r$-band (Fig. 10, upper panel) compared to the $4.5 \mu \mathrm{m}$-band (Fig. 10, bottom panel) is probably related to the lower nuclear extinction in the IR than in the optical bands. Red stars represent the X-ray sources identified as stars based on optical information: XID9, XID63, XID146, XID147, and XID162.

Most of the Chandra source counterparts have been spectroscopically observed with the LBT Multi-Object Double CCD Spectrograph (MODS) and the Large Binocular Telescope Nearinfrared Spectroscopic Utility with Camera and Integral Field Unit for Extragalactic Research (LUCI) for a total of $52 \mathrm{~h}(16 \mathrm{~h}$ with LUCI and $36 \mathrm{~h}$ with MODS) to measure their redshifts. The data reduction and analysis are in progress and the derived properties will be released in the near future (Mignoli et al., in prep.). We will also use the dense multi-band coverage in the J1030 field to derive the photometric redshifts of the X-ray sources (Marchesi et al., in prep.). Photometric redshifts will be used whenever the optical/NIR spectroscopy is missing.

\subsection{Main catalog description}

We present the main Chandra source catalog in Table 4. The details of the table columns are given below.

- Column 1: Source sequence number (XID).

- Columns 2 and 3: RA and Dec of the X-ray source, respectively. These positions were computed through the $\mathrm{AE}$ "CHECK_POSITIONS" procedure (Sect. 3.1). In the catalog we report the centroid position derived from the full band. For sources not detected in the full-band we used the centroid positions derived either from the soft or from the hard band.

- Column 4: Positional error on the source centroid. This was computed as $\sigma=\mathrm{PSF}_{\text {Radius }} / \sqrt{C}$ (Puccetti et al. 2009), where $C$ are the net, background-subtracted, counts computed by AE, and $\mathrm{PSF}_{\text {Radius }}$ is the $90 \%$ encircled energy radius (at $E=1.4 \mathrm{keV}$ ) given by Eq. (1) of Hickox \& Markevitch (2006).

- Column 5: Off-axis angle in arcminutes computed as the angular distance between the position of the X-ray source and the average aim point of the J1030 field (10:30:27.50, $+05: 24: 54.0)$.

- Column 6: Effective exposure time in kiloseconds taken from the full-band exposure map.

- Column 7-9: Hardness ratio computed with Eq. (6) and relative errors. Errors are computed at the $1 \sigma$ level following the method described in Lyons (1991). For display purposes, these three columns are grouped in Col. 7 of Table 4.

- Columns 10-18: Net counts and relative errors computed by $\mathrm{AE}$ in the full $(F)$, soft $(S)$, and hard $(H)$ bands, respectively. Errors are computed according to Tables 1 and 2 of Gehrels (1986) and correspond to the $1 \sigma$ level in Gaussian statistics. For those sources that are not detected in a given band, we provide upper limits at the $3 \sigma$ confidence level (see Sect. 4.1). For display purposes, each band net counts and errors are grouped
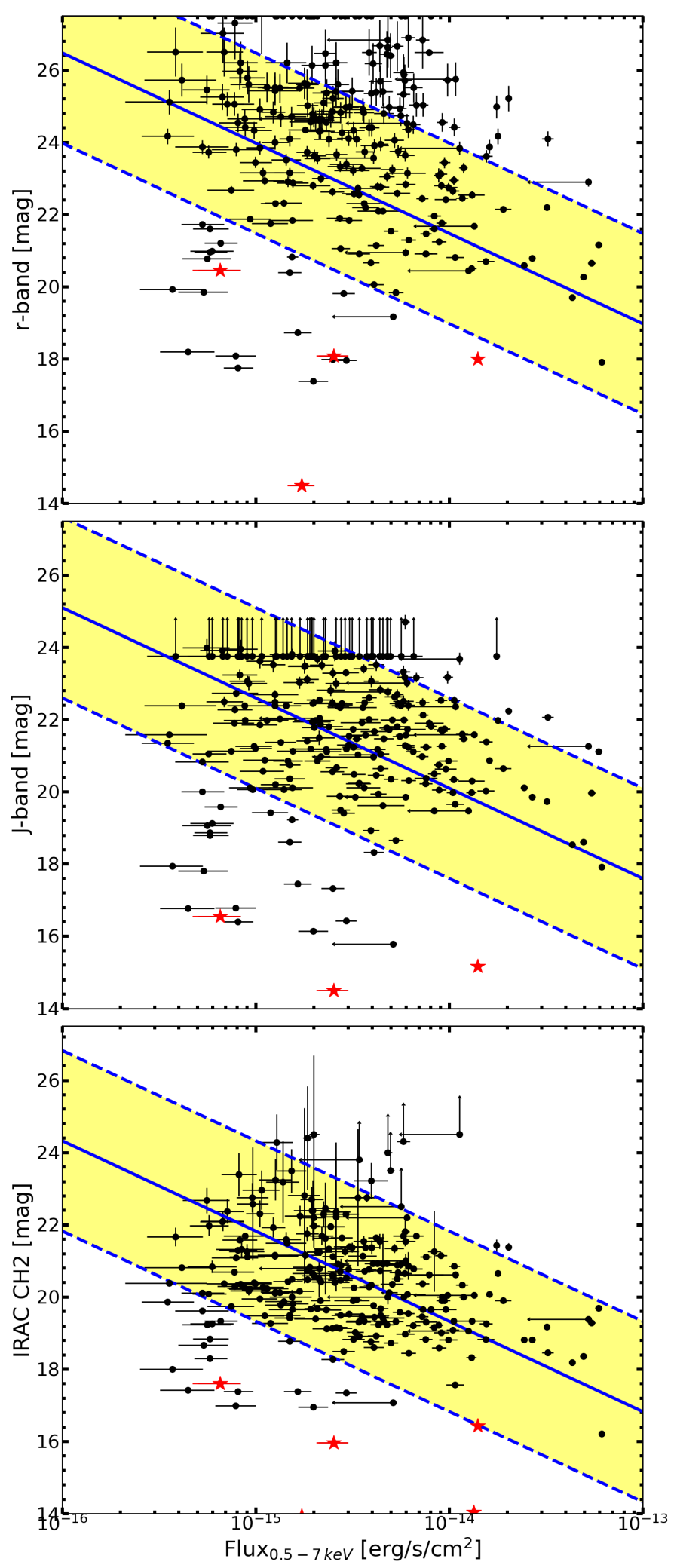

Fig. 10. X-ray full-band flux vs. $r$-band (top panel), $J$-band (middle panel), and $4.5 \mu \mathrm{m}$-band (bottom panel) $\mathrm{AB}$ magnitudes. Black dots represent the main catalog sources, while red stars represent the known stars of our catalog. The blue diagonal dashed lines show constant X-ray to $r-, J-$, or $4.5 \mu \mathrm{m}$-band flux ratios $\log \left(f_{\mathrm{X}} / f_{\text {opt }}\right)=-1,+1$, while the blue diagonal solid line shows $\log \left(f_{\mathrm{X}} / f_{\text {opt }}\right)=0$. The yellow shaded region highlights the area between the blue diagonal dashed lines that, for the optical bands, represents the "classic locus" of unobscured AGNs. 
Table 4. Chandra source catalog.

\begin{tabular}{lccccccccc}
\hline \hline $\begin{array}{l}\text { XID } \\
(1)\end{array}$ & $\begin{array}{c}\text { RA } \\
(2)\end{array}$ & $\begin{array}{c}\text { Dec } \\
(3)\end{array}$ & $\begin{array}{c}\text { Pos err ["] } \\
(4)\end{array}$ & $\begin{array}{c}\text { Off-axis ['] } \\
(5)\end{array}$ & $\begin{array}{c}\text { Exposure [ks] } \\
(6)\end{array}$ & $\begin{array}{c}\text { HR } \\
(7)\end{array}$ & $\begin{array}{c}F \\
(8)\end{array}$ & $\begin{array}{c}S \\
(9)\end{array}$ & $\begin{array}{c}H \\
(10)\end{array}$ \\
\hline 1 & $10: 30: 24.96$ & $+05: 19: 09.40$ & 0.27 & 5.8 & 391.9 & $-0.12_{-0.08}^{+0.08}$ & $251.7_{-16.3}^{+17.3}$ & $138.6_{-11.9}^{+13.0}$ & $113.0_{-11.1}^{+12.1}$ \\
2 & $10: 30: 32.82$ & $+05: 19: 28.80$ & 0.14 & 5.6 & 420.1 & $-0.01_{-0.04}^{+0.04}$ & $827.0_{-29.0}^{+30.0}$ & $416.1_{-20.5}^{+21.5}$ & $420.0_{-20.7}^{+21.8}$ \\
3 & $10: 30: 23.77$ & $+05: 20: 30.23$ & 0.24 & 4.5 & 425.4 & $-0.04_{-0.08}^{+0.08}$ & $164.0_{-13.1}^{+14.1}$ & $84.7_{-9.3}^{+10.4}$ & $79.3_{-9.2}^{+10.2}$ \\
4 & $10: 30: 30.11$ & $+05: 21: 05.96$ & 0.15 & 3.9 & 428.4 & $-0.15_{-0.07}^{+0.07}$ & $259.4_{-16.2}^{+17.2}$ & $147.6_{-12.2}^{+13.2}$ & $109.9_{-10.7}^{+11.7}$ \\
5 & $10: 30: 22.19$ & $+05: 22: 00.79$ & 0.33 & 3.2 & 331.3 & $-0.32_{-0.19}^{+0.19}$ & $36.6_{-6.2}^{+7.3}$ & $23.4_{-4.9}^{+6.0}$ & $12.2_{-3.7}^{+4.8}$ \\
\hline
\end{tabular}

Notes. A complete version of this table with all the 256 sources and properties listed in Sect. 4.3 is available at the CDS.

in Cols. 8-10 for the full, soft, and hard band, respectively, of Table 4.

- Columns 19-21: Binomial no-source probability $P_{\mathrm{B}}$ computed by $\mathrm{AE}$ in the full, soft, and hard bands. Only sources with $P_{\mathrm{B}}<2 \times 10^{-4}$ in at least one band are included in the catalog.

- Columns 22-30: Aperture-corrected X-ray fluxes and relative errors in the full, soft, and hard bands, respectively, while for undetected sources we report $3 \sigma$ upper limits. Fluxes and relative errors were computed from the net rates and relative errors assuming that the full-band spectra of the X-ray sources are power-laws modified by only Galactic absorption with effective power-law photon indices derived from their HRs. For the sources not detected in the soft and hard bands, we assumed a spectral power-law with $\Gamma=1.4$ modified by Galactic absorption.

- Columns 31 and 32: RA and Dec, respectively, of the optical/IR counterpart. When available, we provide the centroid position from the $z$-band catalog, and otherwise we provide the position in other bands in the following order of priority: $J$-band, $r$-band, or $4.5 \mu \mathrm{m}$-band centroid.

- Column 33: Positional offset between the X-ray source and optical counterpart in arcsec.

- Columns 34-37: Counterpart magnitude AUTO in the $r$, $z, J$, and $4.5 \mu \mathrm{m}$ bands, respectively. The reported limits for the undetected counterparts correspond to the limiting AB magnitudes of the corresponding optical/NIR/MIR catalog.

- Columns 38-41: Counterpart magnitude errors in the $r, z$, $J$, and $4.5 \mu \mathrm{m}$ bands, respectively.

- Column 42: Flag providing info on the likelihood of the counterparts: -1 for X-ray sources with no counterpart in any band, 0 for sources with a sub-threshold counterpart, 1 for sources with unique counterpart above likelihood threshold, 2 for sources with two counterparts above threshold (for which we report the counterpart with the highest LR).

- Column 43: Flag notes for the single XID sources: 0 for sources with no morphological information, 1 for sources that have a star as optical/NIR/MIR counterpart based on the optical information, 2 for sources that appear as X-ray extended sources.

The catalog with all the information reported above is publicly available ${ }^{3}$.

\section{Cumulative $\log (N)-\log (S)$ of the $\mathrm{J} 1030$ field}

Finally, we computed the cumulative number of sources, $N(>S)$, brighter than a given flux $(S)$ in each X-ray band. To this aim, we computed the sky coverage (i.e., the sky area $\Omega$ covered as a function of the flux limit) of the J1030 field to correct the incompleteness of our catalog. We computed our sky-coverage by dividing the number of output sources flagged as "good" in

\footnotetext{
3 http://www. oabo.inaf.it/ LBTz6/1030/chandra_1030
}

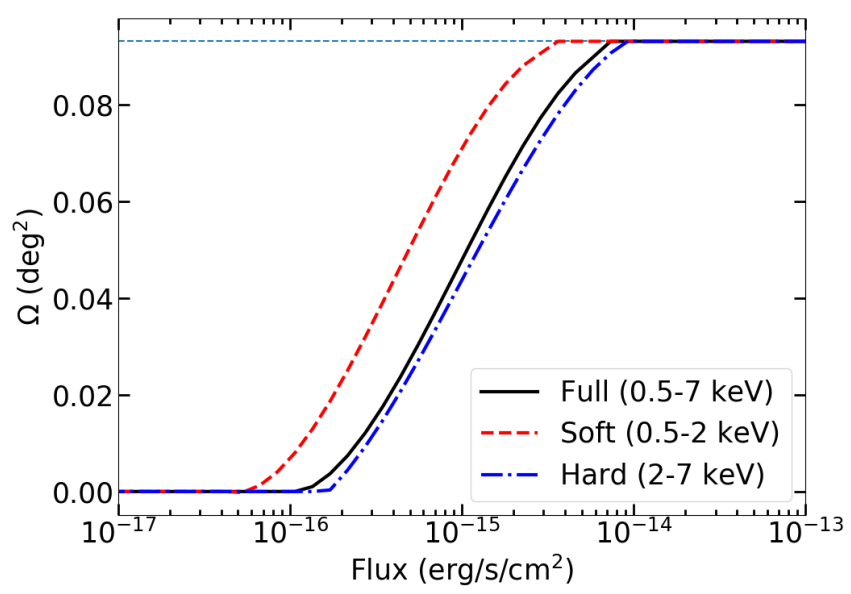

Fig. 11. Sky coverage (i.e., sky area vs. flux limit relation) in the full (black solid line), soft (red dashed line), and hard (blue dash-dotted line) bands derived from our simulations. The horizontal cyan dotted line represents the total geometric area of our J1030 field ( $\left.335 \mathrm{arcmin}^{2}\right)$.

our simulations by the number of input sources as a function of input flux and then multiplying for the total geometric area of the J1030 field covered by Chandra. The sky-coverage values were then fitted with a spline to obtain a smooth monotonically increasing function. The sky coverage in the full, soft, and hard bands are plotted in Fig. 11. The derived flux limits over the central $\sim 1 \operatorname{arcmin}^{2}$ region are $\sim 3 \times 10^{-16}, 6 \times 10^{-17}$, and $2 \times$ $10^{-16} \mathrm{erg} \mathrm{cm}^{-2} \mathrm{~s}^{-1}$ in the full, soft, and hard bands, respectively, making the deep Chandra survey in the SDSS J1030+0524 field the fifth deepest X-ray survey field achieved so far (see Fig. 1 for area-flux curve comparison with other surveys). Once the sky coverage is known, the cumulative source number was computed using the equation:

$N(>S)=\sum_{i=1}^{N_{\mathrm{S}}} \frac{1}{\Omega_{i}} \operatorname{deg}^{-2}$,

where $N_{\mathrm{S}}$ is the total number of detected sources in the field with fluxes higher than $S$, and $\Omega_{i}$ is the sky coverage associated with the flux of the $i$ th source.

The three $\log (N)-\log (S)$ relations in the three X-ray bands are reported in Fig. 12. The red points represent the cumulative number of sources of our $\mathrm{J} 1030$ field, while the $\log (N)-\log (S)$ of our mock catalog are shown as the blue dot-dashed line. For comparison, we also plotted the $\log (N)-\log (S)$ relations found in the $7 \mathrm{Ms}$ (magenta line) Chandra Deep Field-South by Luo et al. (2017), and the $\log (N)-\log (S)$ relation (green solid line) found in the COSMOS field by Civano et al. (2016). From Fig. 12 we conclude that the $\log (N)-\log (S)$ relations derived in the $\mathrm{J} 1030$ 

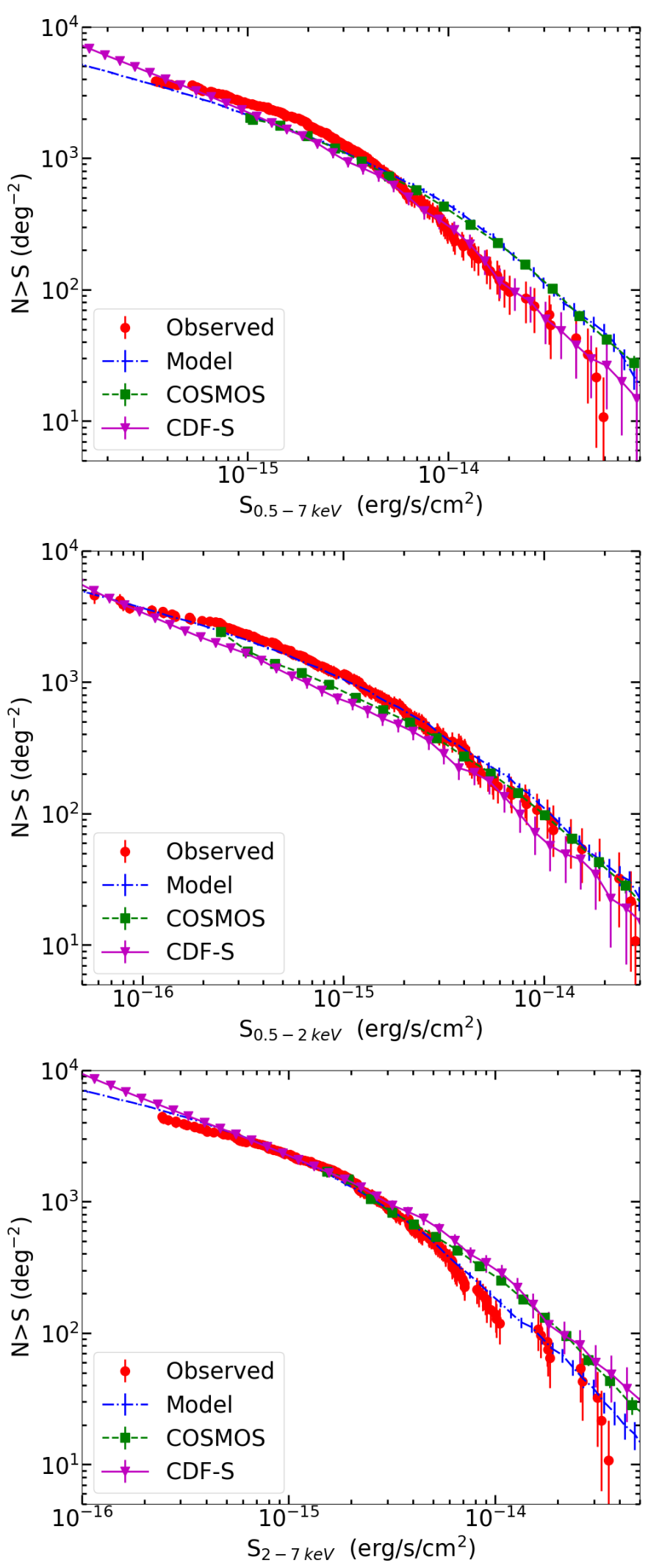

Fig. 12. Cumulative number counts (number of sources brighter than a given flux) for the main source catalog (red dots) in the full (top), soft (middle), and hard (bottom) bands. The blue dot-dashed line represents the cumulative number of sources from our mock catalog. For comparison, we plot the $\log (N)-\log (S)$ relations found in the 7 Ms Chandra Deep Field-South by Luo et al. (2017, magenta line), and in the COSMOS field by Civano et al. (2016, green dotted line). field are in general agreement with those from the literature. Besides cosmic variance, we caution that some of the differences among the $\log (N)-\log (S)$ seen in Fig. 12 could be produced by systematic uncertainties in the different methods used to derive the sky coverage and the individual source fluxes in the various surveys.

\section{Summary}

Here, we present the X-ray source catalog for the deep Chandra survey in the SDSS J1030+0524 field centered on a region that shows the clearest evidence to date of an overdensity around a $z \sim 6$ and an overdensity of galaxies at $z=1.7$. This field has been observed with ten Chandra pointings for a total exposure time of $\sim 479 \mathrm{ks}$ and covers an area of $335 \mathrm{arcmin}^{2}$. Furthermore, the J1030 field is part of the Multiwavelength Yale-Chile survey, and has been entirely observed by Spitzer IRAC, LBT/LBC ( $r$, and $z$ bands), and CHFT/WIRCam ( $J$-band), making J1030 a legacy field for the study of large scale structures around distant accreting SMBHs. Our main results are the following:

- The Chandra source catalog contains 256 X-ray sources that were detected in at least one X-ray band (full, soft, and hard) by wavdetect with a threshold of $10^{-4}$, and filtered by $\mathrm{AE}$ with a binomial probability threshold of $2 \times 10^{-4}$. We assess the binomial probability threshold by producing three X-ray simulations that mirror our Chandra observation, obtaining a completeness of $95 \%$ (full band), $97 \%$ (soft band), and $91 \%$ (hard band), while the reliability levels are $95 \%$ (full band), 96\% (soft band), and 95\% (hard band).

- We achieved X-ray flux limits over the central $\sim 1 \operatorname{arcmin}^{2}$ region of $\sim 3 \times 10^{-16}, 6 \times 10^{-17}$, and $2 \times 10^{-16} \mathrm{erg} \mathrm{cm}^{-2} \mathrm{~s}^{-1}$ in the full, soft, and hard bands, respectively, making the J1030 Chandra field the fifth deepest X-ray survey in existence, after the CDF-S and the CDF-N surveys, the AEGIS-X survey, and the SSA22 survey.

- Based on the multi-band observations of this field, including $r$ and $z$ band data from LBT/LBC, $J$-band imaging from the CFHT/WIRCam, and $4.5 \mu \mathrm{m}$ from Spitzer IRAC, we used a likelihood ratio analysis to associate optical/IR counterparts for $252(98.4 \%)$ of the $256 \mathrm{X}$-ray sources, with an estimated $95 \%$ reliability.

- Finally, we computed the cumulative number of sources in each X-ray band finding that it is in general agreement with both our simulations and those from the CDF-S, the CDF-N, and COSMOS fields.

Acknowledgements. The scientific results reported in this article are based on observations made by the Chandra X-ray Observatory. We acknowledge the referee for a prompt and constructive report. We acknowledge financial contribution from the agreement ASI-INAF n. 2017-14-H.O. We thank P. Broos for providing great support for the analysis of our simulations with AE, and H. M. Günther for the support provided for using MARX. We also thank B. Luo for providing us the $\log (N)-\log (S)$ of the $7 \mathrm{Ms}$ CDF-S. FV acknowledges financial support from CONICYT and CASSACA through the Fourth call for tenders of the CAS-CONICYT Fund, and CONICYT grants Basal-CATA AFB-170002. DM and MA acknowledge support by grant number NNX16AN49G issued through the NASA Astrophysics Data Analysis Program (ADAP). Further support was provided by the Faculty Research Fund (FRF) of Tufts University.

\section{References}

Annunziatella, M., Marchesini, D., Stefanon, M., et al. 2018, PASP, 130, 124501

Balmaverde, B., Gilli, R., Mignoli, M., et al. 2017, A\&A, 606, A23

Bennett, C. L., Larson, D., Weiland, J. L., et al. 2013, ApJS, 208, 20 
Broos, P., Townsley, L., Getman, K., \& Bauer, F. 2012, Astrophysics Source Code Library [record ascl:1203.001]

Brusa, M., Comastri, A., Daddi, E., et al. 2005, A\&A, 432, 69

Brusa, M., Zamorani, G., Comastri, A., et al. 2007, ApJS, 172, 353

Brusa, M., Civano, F., Comastri, A., et al. 2010, ApJ, 716, 348

Cappelluti, N., Hasinger, G., Brusa, M., et al. 2007, ApJS, 172, 341

Cappelluti, N., Brusa, M., Hasinger, G., et al. 2009, A\&A, 497, 635

Cappelluti, N., Li, Y., Ricarte, A., et al. 2017, ApJ, 837, 19

Ciliegi, P., Zamorani, G., Hasinger, G., et al. 2003, A\&A, 398, 901

Civano, F., Elvis, M., Brusa, M., et al. 2012, ApJS, 201, 30

Civano, F., Marchesi, S., Comastri, A., et al. 2016, ApJ, 819, 62

Comastri, A., Ranalli, P., Iwasawa, K., et al. 2011, A\&A, 526, L9

Davis, J. E., Bautz, M. W., Dewey, D., et al. 2012, in Space Telescopes an Instrumentation 2012: Ultraviolet to Gamma Ray, Proc. SPIE, 8443, 84431A De Rosa, G., Decarli, R., Walter, F., et al. 2011, ApJ, 739, 56

Fan, X., Narayanan, V. K., Lupton, R. H., et al. 2001, AJ, 122, 2833

Freeman, P. E., Kashyap, V., Rosner, R., \& Lamb, D. Q. 2002, ApJS, 138, 185

Gawiser, E., van Dokkum, P. G., Herrera, D., et al. 2006, ApJS, 162, 1

Gehrels, N. 1986, ApJ, 303, 336

Gilli, R., Comastri, A., \& Hasinger, G. 2007, A\&A, 463, 79

Gilli, R., Su, J., Norman, C., et al. 2011, ApJ, 730, L28

Gilli, R., Mignoli, M., Peca, A., et al. 2019, A\&A, 632, A26

Goulding, A. D., Forman, W. R., Hickox, R. C., et al. 2012, ApJS, 202, 6

Hickox, R. C., \& Markevitch, M. 2006, ApJ, 645, 95

Kim, S., Stiavelli, M., Trenti, M., et al. 2009, ApJ, 695, 809
Kocevski, D. D., Hasinger, G., Brightman, M., et al. 2018, ApJS, 236, 48 Kurk, J. D., Walter, F., Fan, X., et al. 2007, ApJ, 669, 32

LaMassa, S. M., Urry, C. M., Cappelluti, N., et al. 2016, ApJ, 817, 172 Lehmer, B. D., Alexander, D. M., Chapman, S. C., et al. 2009, MNRAS, 400, 299

Lehmer, B. D., Xue, Y. Q., Brandt, W. N., et al. 2012, ApJ, 752, 46 Luo, B., Brandt, W. N., Xue, Y. Q., et al. 2010, ApJS, 187, 560

Luo, B., Brandt, W. N., Xue, Y. Q., et al. 2017, ApJS, 228, 2

Lyons, L. 1991, A Practical Guide to Data Analysis for Physical Science Students (Cambridge, UK: Cambridge University Press), 107

Marchesi, S., Civano, F., Salvato, M., et al. 2016, ApJ, 827, 150

Menzel, M. L., Merloni, A., Georgakakis, A., et al. 2016, MNRAS, 457, 110

Morselli, L., Mignoli, M., Gilli, R., et al. 2014, A\&A, 568, A1

Murray, S. S., Kenter, A., Forman, W. R., et al. 2005, ApJS, 161, 1

Nandra, K., Laird, E. S., Aird, J. A., et al. 2015, ApJS, 220, 10

Nanni, R., Gilli, R., Vignali, C., et al. 2018, A\&A, 614, A121

Norman, C., Ptak, A., Hornschemeier, A., et al. 2004, ApJ, 607, 721

Puccetti, S., Vignali, C., Cappelluti, N., et al. 2009, ApJS, 185, 586

Quadri, R., Marchesini, D., van Dokkum, P., et al. 2007, AJ, 134, 1103

Ranalli, P., Comastri, A., \& Setti, G. 2005, A\&A, 440, 23

Stiavelli, M., Djorgovski, S. G., Pavlovsky, C., et al. 2005, ApJ, 622, L1

Sutherland, W., \& Saunders, W. 1992, MNRAS, 259, 413

Xue, Y. Q., Luo, B., Brandt, W. N., et al. 2011, ApJS, 195, 10

Xue, Y. Q., Luo, B., Brandt, W. N., et al. 2016, ApJS, 224, 15

Zamorani, G., Mignoli, M., Hasinger, G., et al. 1999, A\&A, 346, 731 


\section{Appendix A: Likelihood-ratio method}

As described in Sect. 3.3, after producing simulations that mirror our Chandra observation of $\mathrm{J} 1030$ and using wavdetect to detect the sources on these simulated fields, we needed a numerical method to disentangle output sources that actually correspond to input sources from those that are spurious detections. To this purpose, we used a LR method to match output with input sources. The LR method we adopted has been used previously to match sources detected at different wavelengths (e.g., Sutherland \& Saunders 1992; Ciliegi et al. 2003; Brusa et al. 2007; Luo et al. 2010), and is available online ${ }^{4}$. For an input-simulated candidate with a flux $f$ at an angular separation $r$ from a given X-ray output, the LR is defined as in Eq. (2):

$\mathrm{LR}=\frac{F(r) q(f)}{n(f)}$.

In Eq. (2) we assumed that $F(r)$ (the probability distribution function of the angular separation) follows a Gaussian distribution (e.g., Zamorani et al. 1999):

$F(r)=\frac{1}{2 \pi \sigma_{\text {pos }}^{2}} \exp \left(\frac{-r^{2}}{2 \sigma_{\text {pos }}^{2}}\right)$,

where $\sigma_{\text {pos }}$ is the $1 \sigma$ positional error of the X-ray detected sources computed as $\sigma_{\text {pos }}=\mathrm{PSF}_{\text {Radius }} / \sqrt{C}$ (Puccetti et al. 2009), $C$ are the net background-subtracted counts computed by $\mathrm{AE}$, and $\mathrm{PSF}_{\text {Radius }}$ is evaluated with the estimate at the $90 \%$ encircled energy radius (at $E=1.4 \mathrm{keV}$ ) at off-axis $(\theta)$ as $\operatorname{PSF}_{\text {Radius }}=1^{\prime \prime}+10^{\prime \prime}\left(\theta / 10^{\prime}\right)^{2}$ (Hickox \& Markevitch 2006).

The flux-dependent surface density of the background sources, $n(f)$, is estimated using our sample of input-simulated sources that are at an angular separation inside an annulus from any of the output-detected sources $\left(r_{\text {in }}=5^{\prime \prime}\right.$ and $r_{\text {out }}=30^{\prime \prime}$; e.g., Luo et al. 2010). Input sources that fall inside the annular regions are considered as background sources.

Here, $q(f)$ is the expected flux distribution of the real counterparts, and is not directly observable. To derive an estimate of $q(f)$, the LR method selects all input sources within $r_{\text {in }}=2^{\prime \prime}$ from any detected source. The flux distribution of these sources is denoted as total $(f)$, which was then background-subtracted to derive:

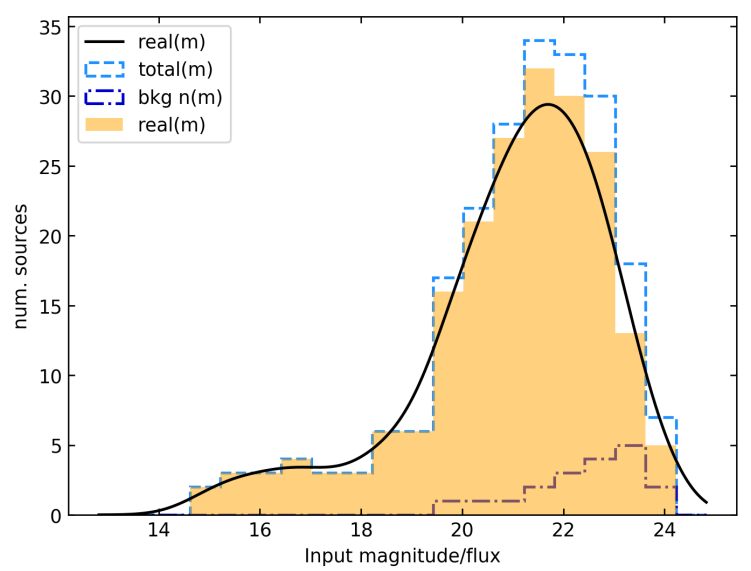

Fig. A.1. Magnitude distribution of the $J$-band counterparts $(q(f)$, black line) and the magnitude distribution of the background sources $(n(f)$, blue dot-dashed line). The cyan dashed line represents the $\operatorname{total}(f)$ (see Appendix A) while the orange shaded area is the $\operatorname{total}(f)$ defined as in Eq. (A.3).

$\operatorname{real}(f)=\operatorname{total}(f)-\pi r_{\mathrm{in}}^{2} N_{\mathrm{out}} n(f)$,

where $N_{\text {out }}$ is the total number of X-ray detected sources.

An example of $\operatorname{real}(f), \operatorname{total}(f), n(f)$, and $q(f)$ distribution for the $J$-band counterparts is reported in Fig. A.1. Due to the magnitude limits of the input catalog, we were only able to detect a fraction $Q$ of all the true counterparts (see Sect. 3.3 for the definition of $Q$ ). Thus the expected flux distribution of the counterparts $q(f)$ is derived by normalizing $\operatorname{real}(f)$ and then multiplying by $Q$ :

$q(f)=\frac{\operatorname{real}(f)}{\sum_{i} \operatorname{real}(f)_{i}} Q$.

Having computed the values of $q(f), n(f)$, and $f(r)$, our LR method calculates LR values for all the input sources within $r_{\mathrm{LR}}=5^{\prime \prime}$ from each output detected source. Postage-stamp images for the X-ray catalog sources are reported in Fig. A.2. The images are color composites of the LBT/LBC $r, z$, and CFHT/WIRCam $J$ bands. In Fig. A.3 we show 17 X-ray sources that, among the four optical/IR band explored, have a counterpart only at $4.5 \mu \mathrm{m}$

\footnotetext{
4 https://github.com/alessandropeca/LYR PythonLikelihoodRatio
} 
R. Nanni et al.: The deep Chandra survey in the SDSS J1030+0524 field
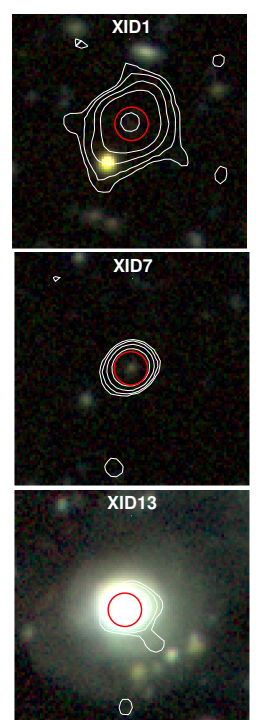

XID19
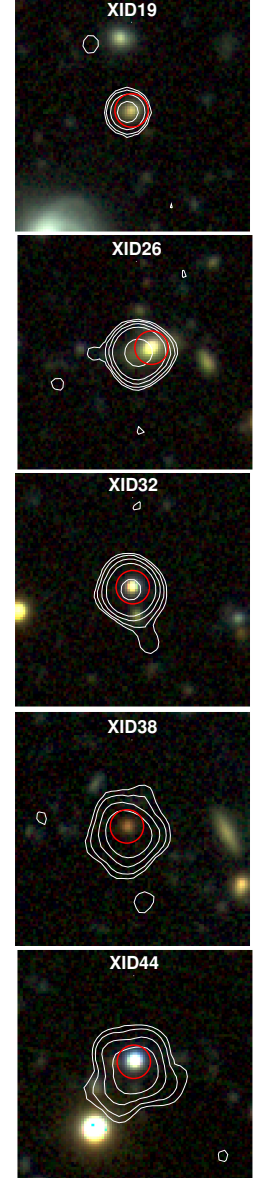

XID50

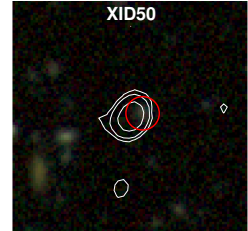

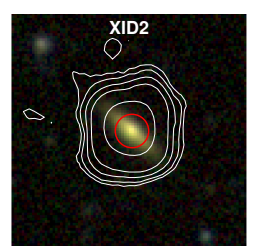
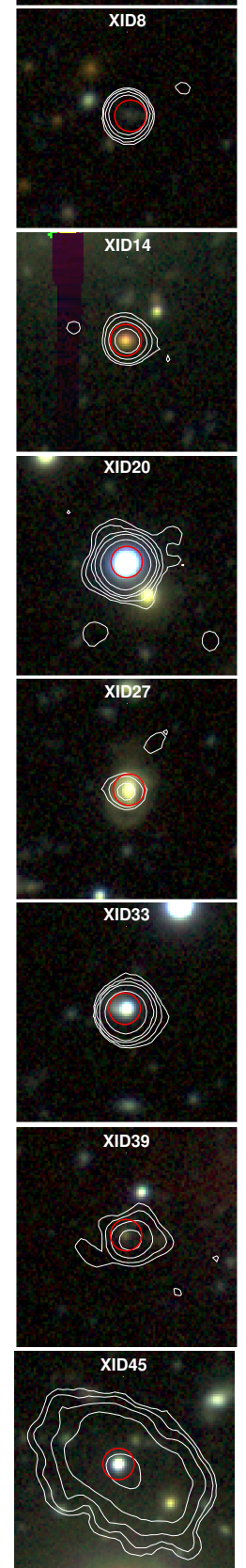

XID51

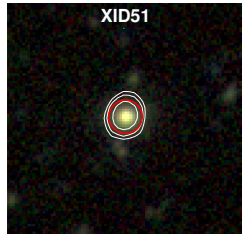

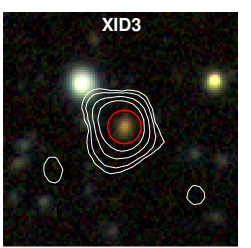
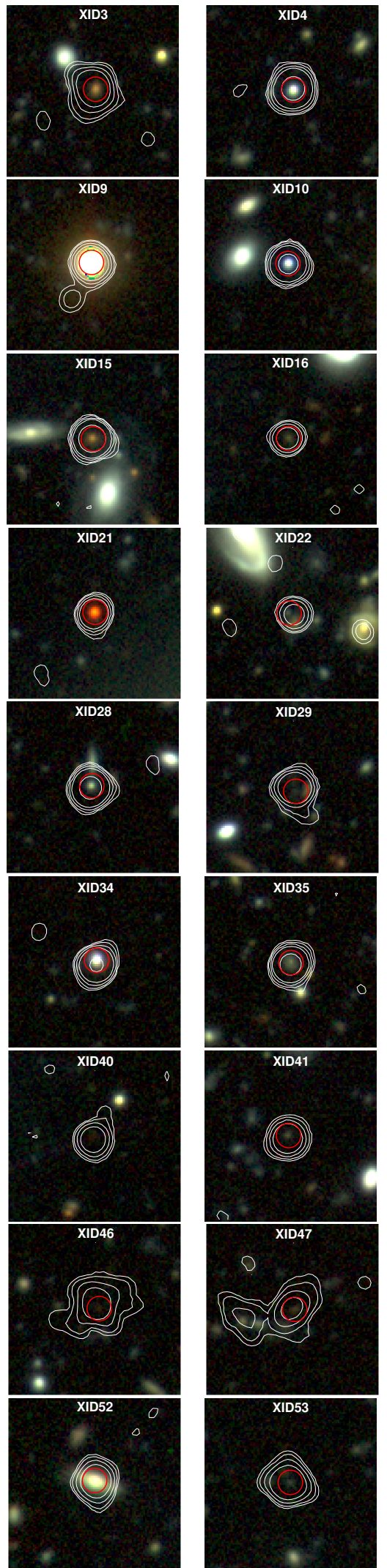

XID53
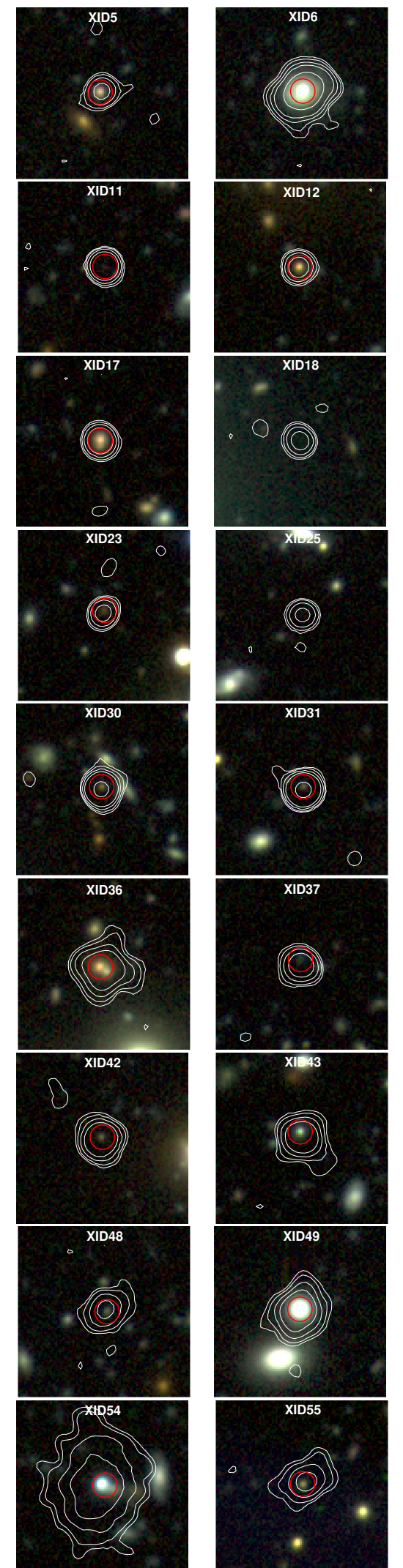

Fig. A.2. Postage-stamp images for the X-ray catalog sources. The images are color composites of the LBT/LBC $r$, $z$, and CFHT/WIRCam $J$ bands. The red circles $\left(r=1.5^{\prime \prime}\right)$ mark the optical/NIR position of the counterpart detected in at least one of the three considered bands $(r, z, J)$, while the white contours show the X-ray full-band contours at different significance levels: $3,5,10$, 20, and $100 \sigma$. Each image is $20^{\prime \prime}$ on a side and is centered on the X-ray centroid. The catalog XID is shown at the top of each image. For $21 \mathrm{X}$-ray sources there is no likely optical/NIR counterpart (the red circle being absent). For 17 of them, we present in Fig. A.3 their IRAC postage-stamp image showing the $4.5 \mu \mathrm{m}$ counterpart. 
A\&A 637, A52 (2020)

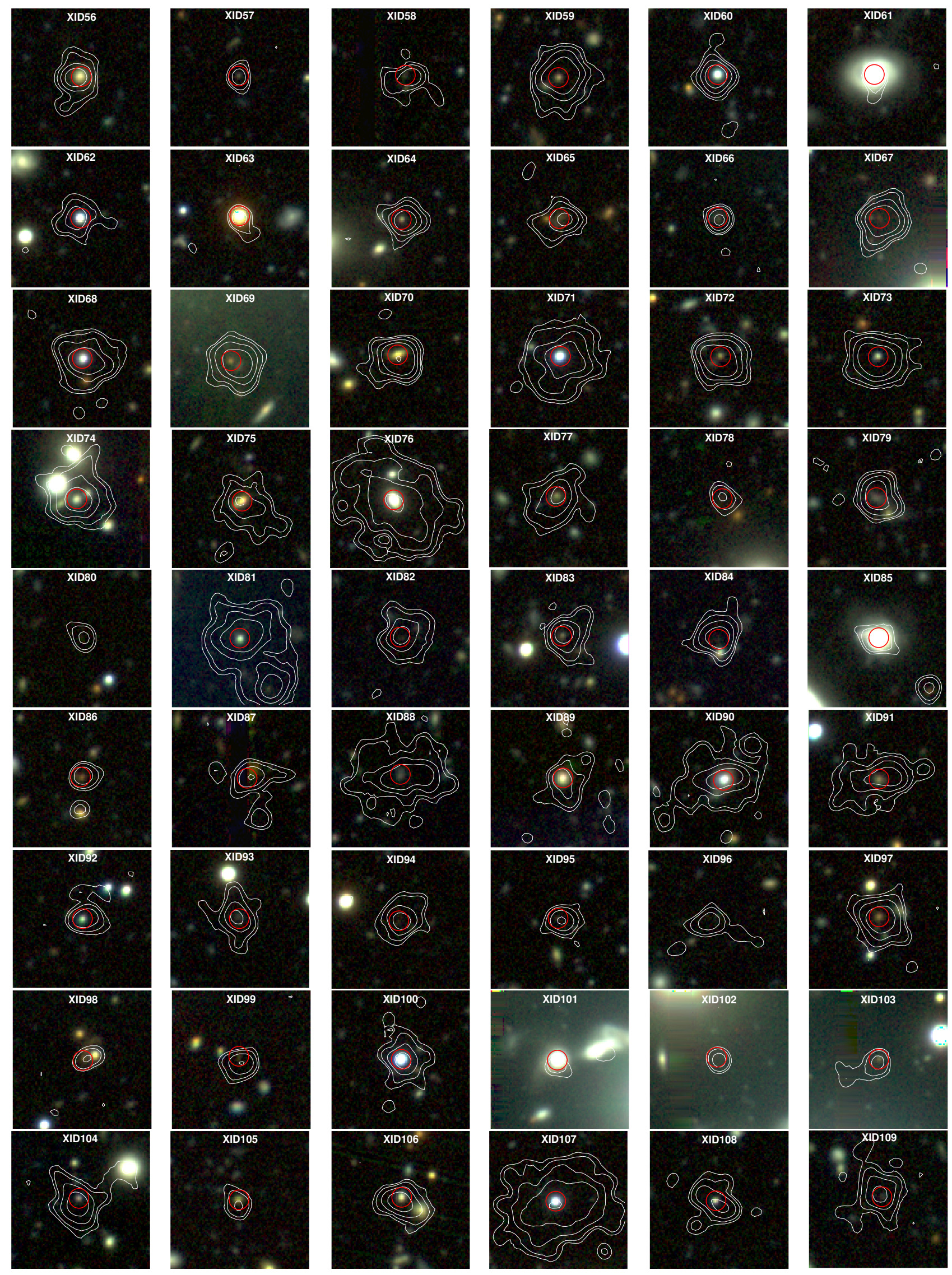

Fig. A.2. continued. 
R. Nanni et al.: The deep Chandra survey in the SDSS J1030+0524 field
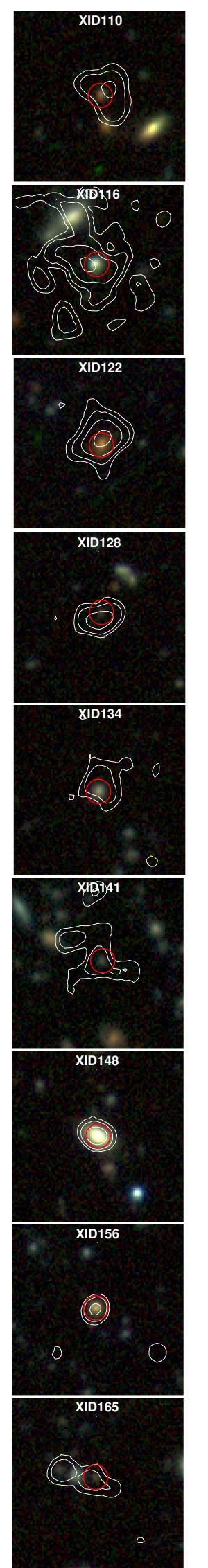

Fig. A.2. continued.
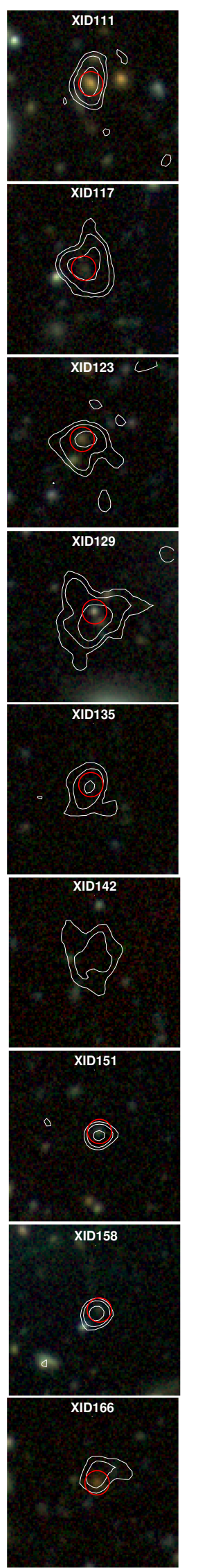
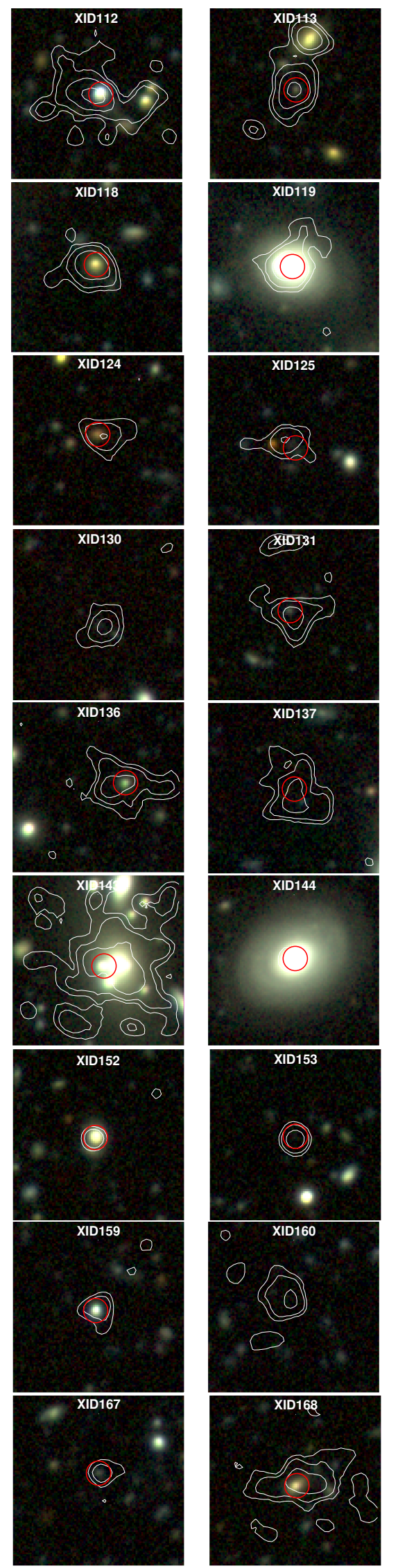
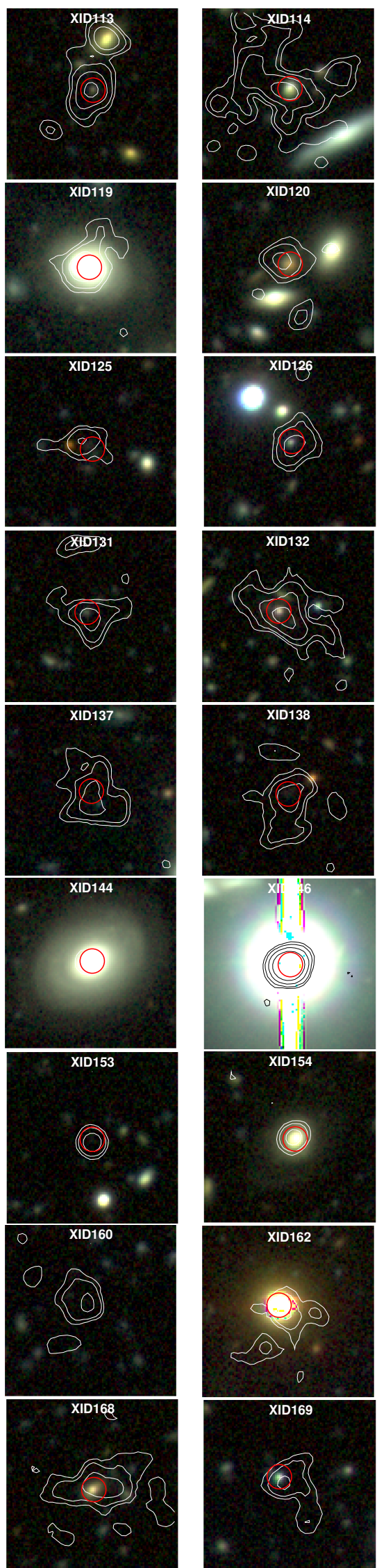
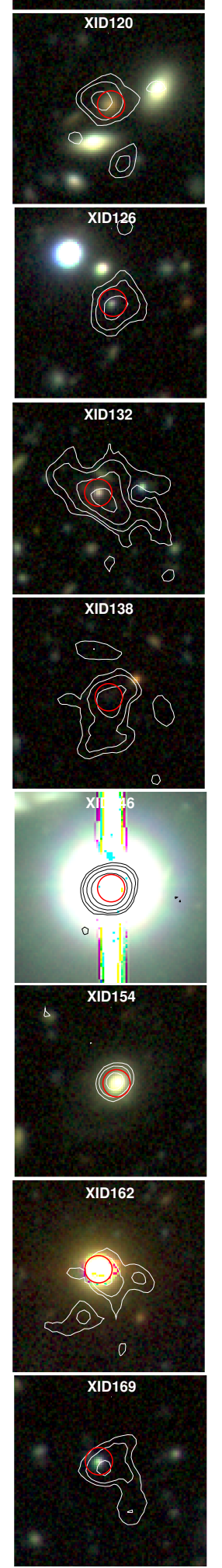
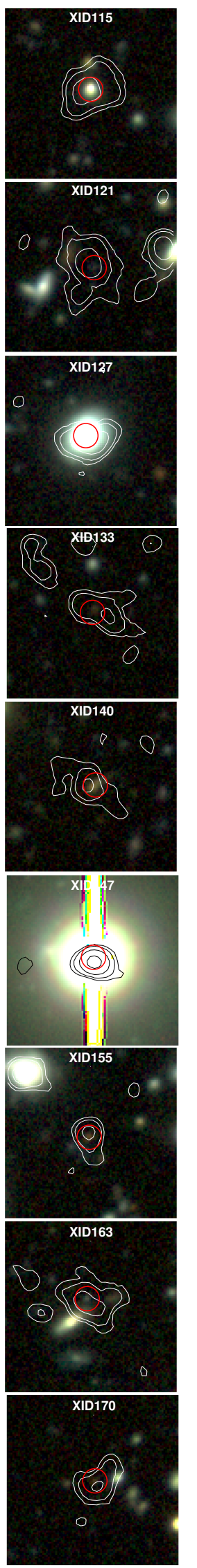
A\&A 637, A52 (2020)

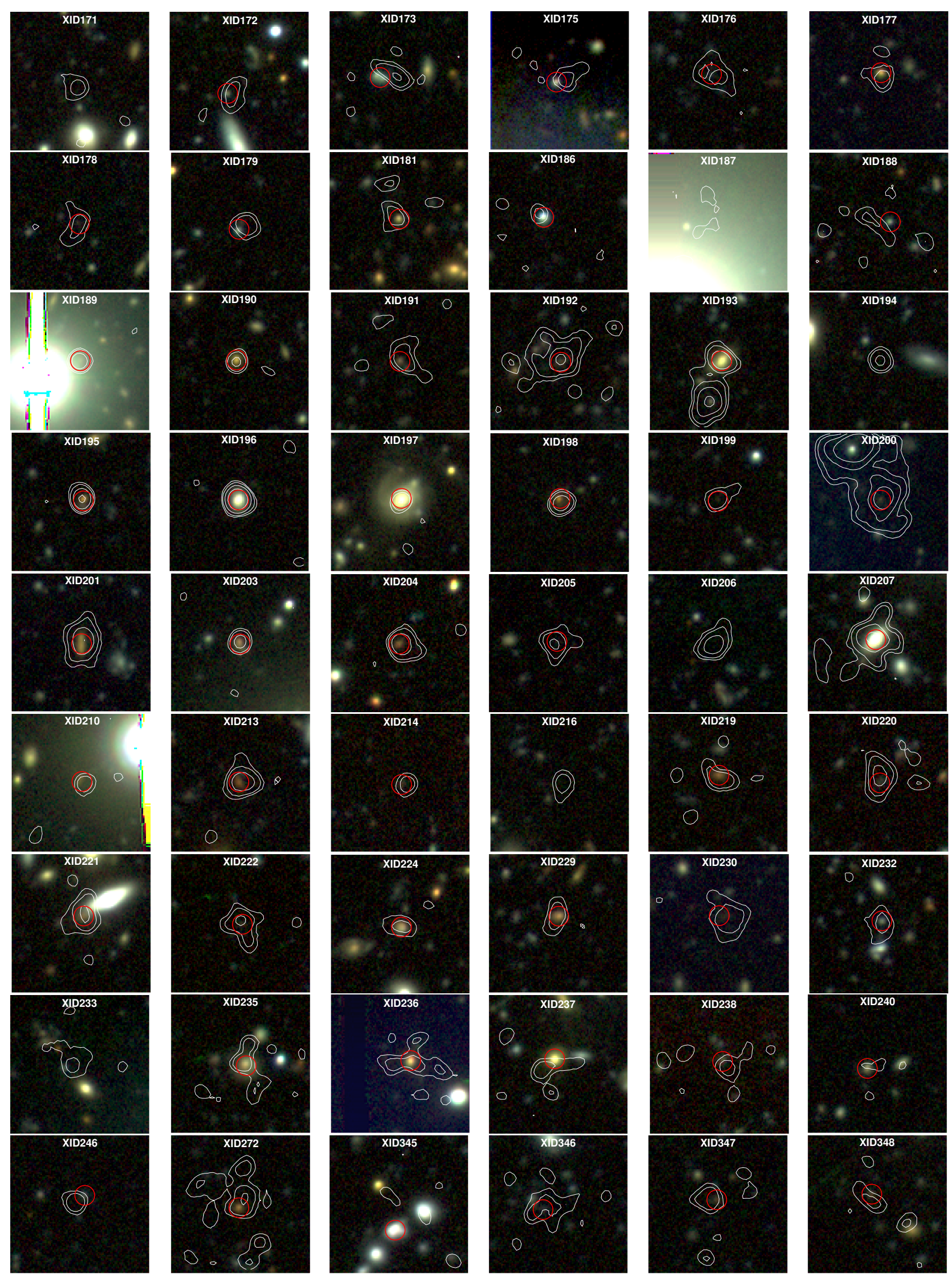

Fig. A.2. continued. 
R. Nanni et al.: The deep Chandra survey in the SDSS J1030+0524 field
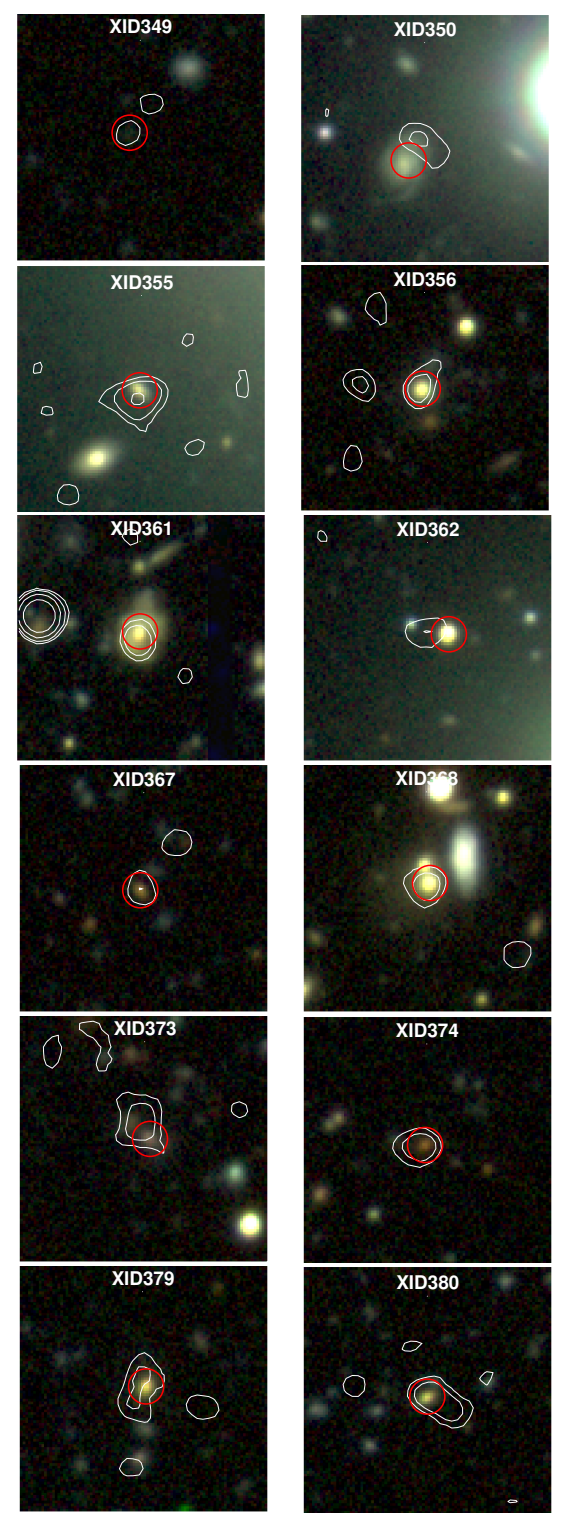

XID385

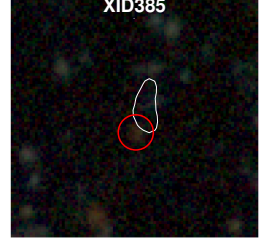

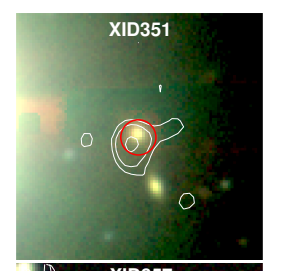
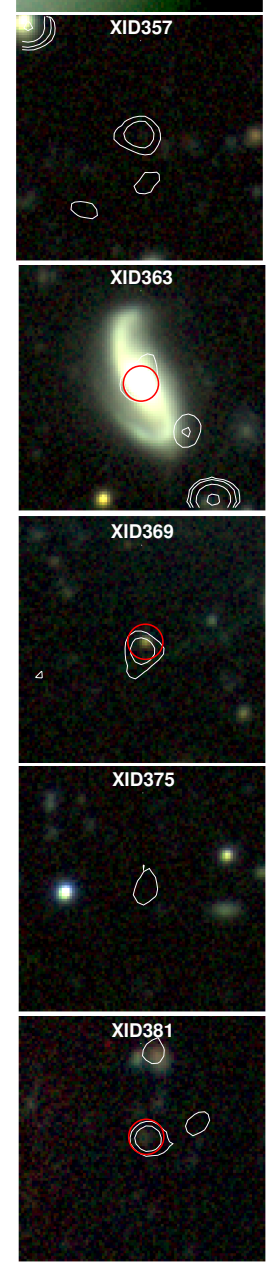

XID386

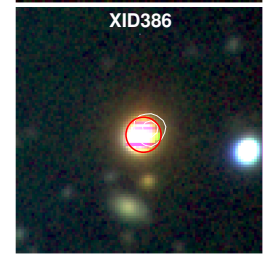

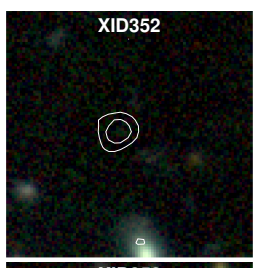
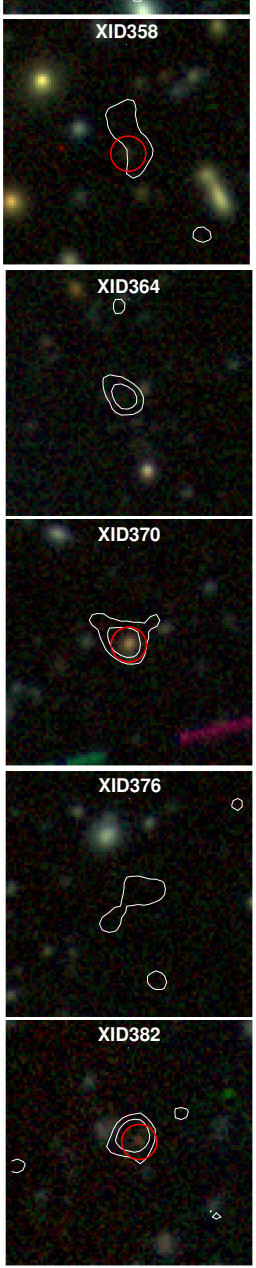

XID387

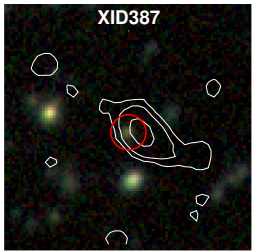

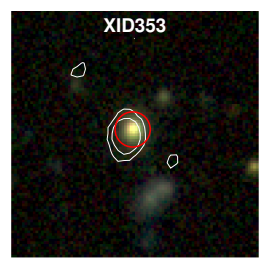

XID359
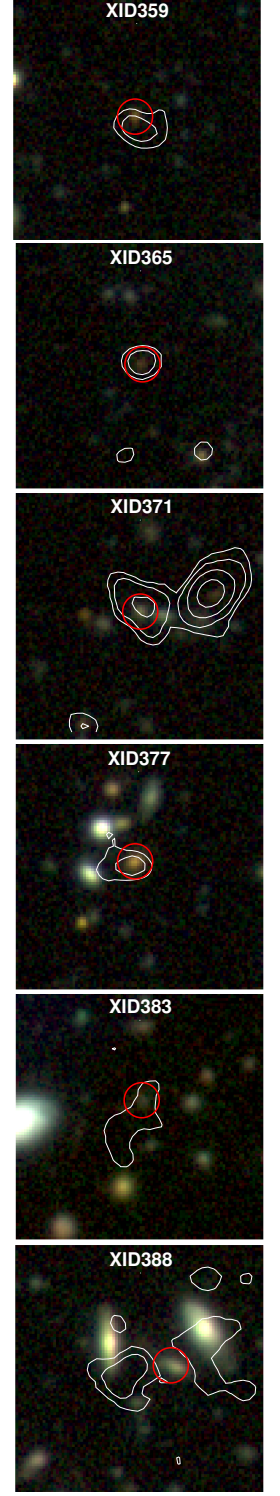

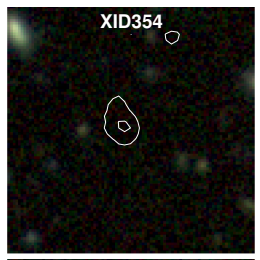

XID360
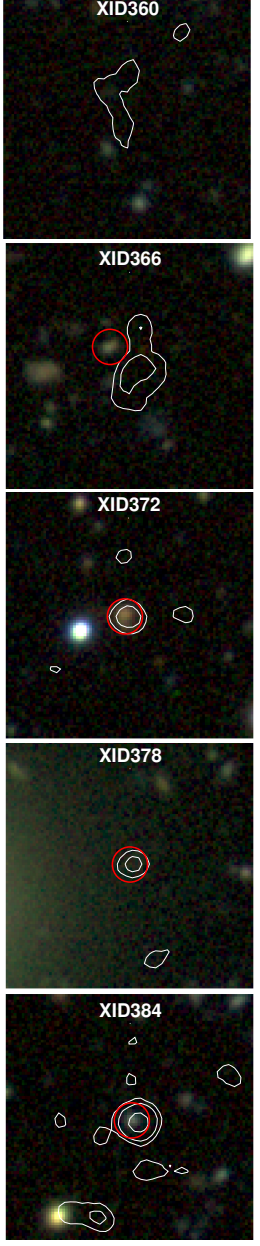

Fig. A.2. continued. 


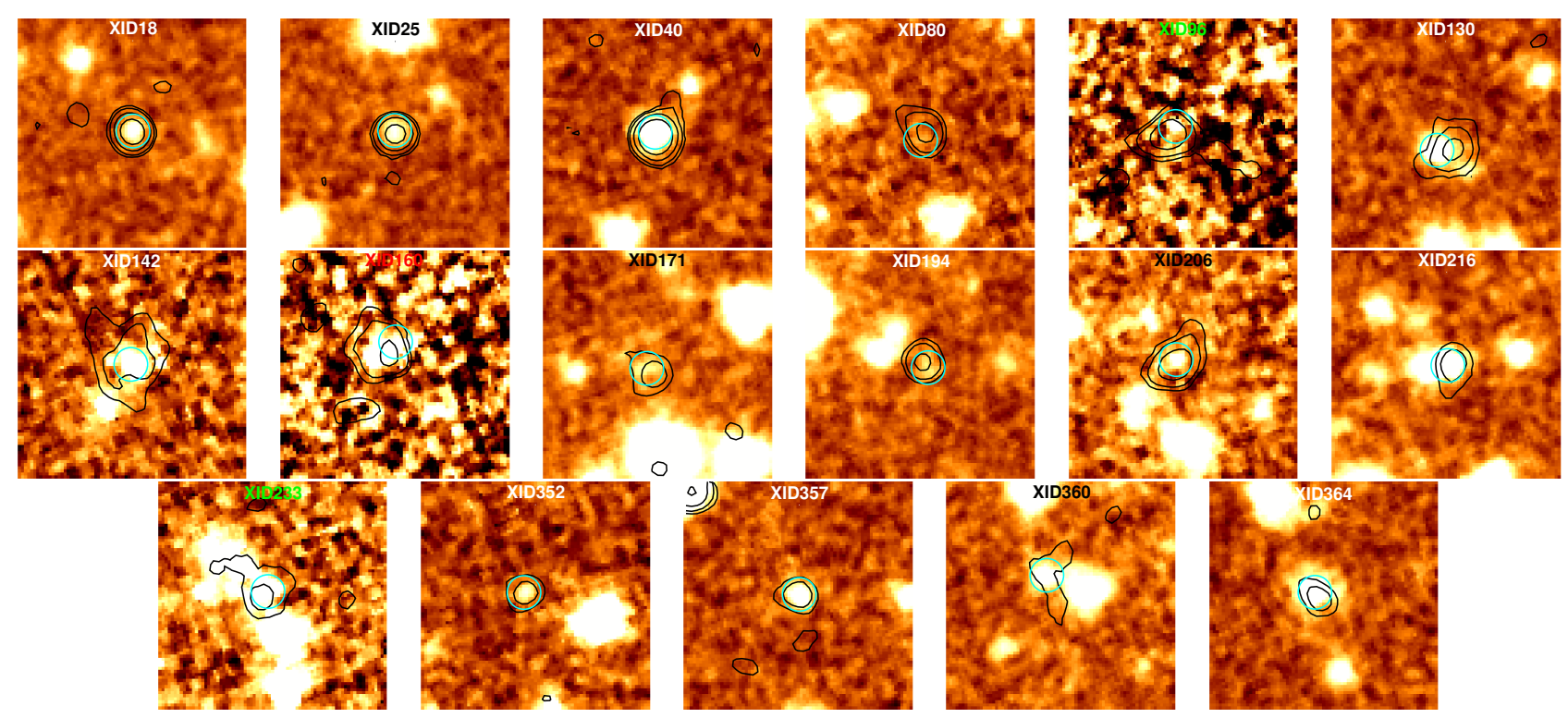

Fig. A.3. Postage-stamp images for the X-ray sources that, among the four optical/IR band explored, have a counterpart only at $4.5 \mu \mathrm{m}$. The cyan circles $\left(r=1.5^{\prime \prime}\right)$ mark the MIR position of the counterpart, while the black contours show the X-ray full-band contours at different significance levels: $3,5,10,20$, and $100 \sigma$. Each image is $20^{\prime \prime}$ on a side and is centered on the X-ray centroid. The catalog XID is shown at the top of each image. 